\title{
Influence of Langmuir circulations on turbulence in the bottom boundary layer of shallow water
}

\author{
Bing-Qing Deng ${ }^{1}$, Zixuan Yang ${ }^{1,2}$, Anqing Xuan $^{1}$ and Lian Shen ${ }^{1} \dagger$ \\ ${ }^{1}$ St. Anthony Falls Laboratory and Department of Mechanical Engineering, University of Minnesota, \\ Minneapolis, Minnesota 55455, USA \\ ${ }^{2}$ State Key Laboratory of Nonlinear Mechanics, Institute of Mechanics, Chinese Academy of Sciences, \\ Beijing 100190, PR China
}

(Received 17 April 2018; revised 9 September 2018; accepted 28 October 2018; first published online 19 December 2018)

Langmuir circulations (LCs) generated by the interaction between wind-driven currents and surface waves can engulf the whole water column in neutrally stratified shallow water and interact with the turbulence in the bottom boundary layer. In this study, we perform a mechanistic study using wall-resolved large-eddy simulations (LES) based on the Craik-Leibovich equations to investigate the effects of LCs on turbulence statistics in the bottom half of shallow water. The highest Reynolds number considered in this paper, $R e_{\tau}=1000$, is larger than the values considered in wall-resolved LES studies of shallow-water Langmuir turbulence reported in literature. The logarithmic layer is diagnosed based on a plateau region in the profile of a diagnostic function. It is found that the logarithmic layer disrupted at $R e_{\tau}=395$ reappears at $R e_{\tau}=1000$, but the von Kármán constant is slightly different from the traditional value 0.41 . To study the effects of LCs on turbulence statistics, LCs are extracted using streamwise averaging. The velocity fluctuations $u_{i}^{\prime}$ are decomposed into a LC-coherent part $u_{i}^{L}$ and a residual turbulence part $u_{i}^{T}$. It is found that the profiles of LC-coherent Reynolds shear stress $-\left\langle u^{L} v^{L}\right\rangle$ obtained at various Reynolds numbers are close to each other in the water-column coordinate $y / h$, with $h$ being the half-water depth. As the Reynolds number (or, by definition, the ratio between the outer and inner length scales) increases, the influence of LCs on the near-bottom momentum transfer is reduced, which is responsible for the reappearance of the logarithmic layer. At all of the Reynolds numbers under investigation, the peaks of $\left\langle u^{L} u^{L}\right\rangle$ are collocated in the water-column coordinate $y / h$, while those of $\left\langle u^{T} u^{T}\right\rangle$ are collocated in the inner-scale coordinate $y /\left(v / u_{\tau}\right)$. Due to the increase in the distance between the peaks of $\left\langle u^{L} u^{L}\right\rangle$ and $\left\langle u^{T} u^{T}\right\rangle$ with the Reynolds number, the profile of $\left\langle u^{\prime} u^{\prime}\right\rangle$ forms a bimodal shape at $R e_{\tau}=700$ and 1000 .

Key words: turbulence simulation, turbulent boundary layers, wave-turbulence interactions

$†$ Email address for correspondence: shen@umn.edu 


\section{Introduction}

The interaction between wind-shear-driven currents and surface water waves generates Langmuir circulations (LCs), manifested by the long windrows (or streaks) extending along the wind direction at the water surface (Leibovich 1983; Smith 2001; Thorpe 2004; Sullivan \& McWilliams 2010; D’Asaro 2014). LCs are named after Irving Langmuir for his pioneering contribution to the investigation of windrows based on a series of flow visualization experiments (Langmuir 1938). He attributed the windrows to the convergent motion between pairs of counter-rotating streamwise vortices in the water. Following the work of Langmuir, many field observations and laboratory experiments were conducted to study the characteristics of LCs in the upper-ocean mixed layer (see, e.g. the reviews by Pollard 1977; Leibovich 1983; Thorpe 2004). The previous studies of LCs in deep water investigated the length scales of windrows, the vertical penetration depth of LCs and the intensities of the upwelling and downwelling motions induced by LCs (Langmuir 1938; Scott et al. 1969; Assaf, Gerard \& Gordon 1971; Smith, Pinkel \& Weller 1987; Weller \& Price 1988; Nepf 1992; Thorpe 1992; Farmer \& Li 1995). It was reported that the strong downwelling and upwelling motions of LCs enhance the vertical mixing and attenuate the mean shear in the mixed layer (Weller \& Price 1988; Nepf 1992; Thorpe 1992; Farmer \& Li 1995). In neutrally stratified shallow water, LCs can engulf the entire water column (Faller \& Caponi 1978; Gargett et al. 2004; Gargett \& Wells 2007; Kukulka, Plueddemann \& Sullivan 2012; Savidge \& Gargett 2017). These large-scale full-depth LCs greatly enhance the vertical mixing of scalar quantities and momentum throughout the water column (Gargett et al. 2004; Dethleff \& Kemepema 2007; Gargett \& Wells 2007; Dierssen et al. 2009; Kukulka et al. 2012; Akan et al. 2013), and have a significant impact on the bottom boundary layer (Tejada-Martínez \& Grosch 2007; Tejada-Martínez et al. 2012).

Craik \& Leibovich (1976) and Leibovich (1977) derived the Craik-Leibovich (C-L) equations to explain the mechanism underlying LCs. The $\mathrm{C}-\mathrm{L}$ equations describe the wave-phase-averaged effect of surface waves on the shear turbulence through a vortex force term in the momentum equations, which depends on the Stokes drift velocity of surface waves and the vorticity of flow field. The derivation of the $\mathrm{C}-\mathrm{L}$ equations was also given in Andrews \& McIntyre (1978), Leibovich (1980) and Holm (1996). Through the stability analysis of the $\mathrm{C}-\mathrm{L}$ equations, it was found that the vertical vorticity generated by the disturbance of the shear-driven current is tilted into the streamwise direction by the shear of the Stokes drift of surface waves to generate streamwise vortices (Craik 1977).

The development of the $\mathrm{C}-\mathrm{L}$ equations makes it possible to perform numerical simulations to study Langmuir turbulence without resolving the wave surface. Skyllingstad \& Denbo (1995) and McWilliams, Sullivan \& Moeng (1997) performed large-eddy simulations (LES) for Langmuir turbulence in the mixed layer based on the C-L equations. McWilliams et al. (1997) pointed out that the turbulent Langmuir number, $L a_{t}=\sqrt{u_{\tau} / u_{s}^{*}}$, is a key dimensionless parameter in Langmuir turbulence that quantifies the relative importance of the wind shear to the $\mathrm{C}-\mathrm{L}$ vortex force related to the Stokes drift of surface waves. Here, $u_{\tau}$ is the surface friction velocity, and $u_{s}^{*}$ is the characteristic value of Stokes drift velocity, defined as the surface Stokes drift in deep-water Langmuir turbulence. As the value of $L a_{t}$ decreases, the LCs are enhanced, characterized by the increase of the variance of vertical velocity fluctuations (Li, Garrett \& Skyllingstad 2005; Harcourt \& D'Asaro 2008). The LCs are also influenced by the parameter $k h$, where $k$ is the wavenumber of the dominant wave and $h$ is the depth of mixed layer. It was found that the location of the maximum 
vertical velocity in deep-water Langmuir turbulence moves towards the water surface as $k h$ increases ( $\mathrm{Li}$ et al. 2005; Harcourt \& D'Asaro 2008). LES based on the C-L equations were also performed to study the deep-water Langmuir turbulence under complex sea conditions, including modelling for wave breaking (Noh, Min \& Raasch 2004; Sullivan, McWilliams \& Melville 2007), swells coexisting with wind seas (McWilliams et al. 2014) and hurricanes (Sullivan et al. 2012).

While the $\mathrm{C}-\mathrm{L}$ equations were solved numerically to study deep-water Langmuir turbulence as reviewed above, for shallow waters, LES based on the $\mathrm{C}-\mathrm{L}$ equations were also conducted to study the influence of full-depth LCs on the turbulence in the bottom, core and surface regions of the neutrally stratified shallow-water column (Tejada-Martínez \& Grosch 2007; Kukulka et al. 2012; Tejada-Martínez et al. 2012; Akan et al. 2013; Tejada-Martínez et al. 2013; Martinat, Grosch \& Gatski 2014; Sinha et al. 2015). It was reported that in shallow-water Langmuir turbulence, the mean shear in the core region reduces and even becomes negative, and the logarithmic layer disappears due to the disruption effect of the LCs (Tejada-Martínez et al. 2012, 2013; Sinha et al. 2015). In comparison with the pure shear-driven turbulence without LCs, the LCs significantly enhance the spanwise velocity fluctuations near the water bottom and the vertical velocity fluctuations in the core region of the water column (Tejada-Martínez \& Grosch 2007; Kukulka et al. 2012; Akan et al. 2013; Martinat et al. 2014; Sinha et al. 2015). The results obtained from the LES show good agreement with the observations of full-depth LCs in an unstratified shallow-water column $(15 \mathrm{~m})$ on the shelf approximately 6-7 $\mathrm{km}$ off the New Jersey coast (Gargett et al. 2004; Gargett \& Wells 2007). The effects of Coriolis force (Grosch \& Gargett 2016), surface heat flux (Gargett et al. 2004; Gargett \& Wells 2007; Gargett \& Grosch 2014; Gargett, Savidge \& Wells 2014; Walker, Tejada-Martínez \& Grosch 2016) and tidal current (Kukulka et al. 2011; Martinat et al. 2011) on shallow-water Langmuir turbulence are also studied through LES and field measurements.

To study the mechanism of the effect of full-depth LCs on turbulence statistics in the bottom boundary layer, wall-resolved LES (i.e. without wall-layer modelling) has been used in the literature to resolve the near-bottom region. Only full-depth LCs can deeply penetrate into the bottom boundary. This is the focus of the present study. Hereafter, full-depth LCs are called LCs for short. In previous studies, wall-resolved LES of shallow-water Langmuir turbulence was carried out at relatively low Reynolds numbers, e.g. $R e_{\tau}=u_{\tau} h / v=395$ and 590 (Tejada-Martínez \& Grosch 2007; Tejada-Martínez et al. 2012). Here, $h$ is the half-water depth, and $v$ is the kinematic viscosity. The disruption of the logarithmic layer implies that the traditional wall-layer modelling based on the logarithmic law of the mean velocity (Cabot \& Moin 2000; Piomelli \& Balaras 2002) is inappropriate. The above conclusion is, however, based on relatively low Reynolds numbers, and needs to be further examined at higher Reynolds numbers. Furthermore, the increase of Reynolds number differentiates the inner and outer length scales. It was found that in canonical wall turbulence at high Reynolds numbers $\left(\operatorname{Re}_{\tau}=O\left(10^{3}-10^{5}\right)\right)$, the length scale separation leads to the emergence of large-scale motions scaled by the outer length scale, which make the shape of Reynolds normal stress profiles different from that at low Reynolds numbers $\left(R e_{\tau}=O\left(10^{2}\right)\right.$ ) (Hoyas \& Jiménez 2006; Marusic, Mathis \& Hutchins 2010; Jiménez 2012). From the above review of previous studies of Langmuir turbulence and canonical wall turbulence, it is understood that wall-resolved LES at higher Reynolds numbers are needed to further study the effects of LCs on the bottom boundary layer. The recent growth in computer power allows us to perform wall-resolved 


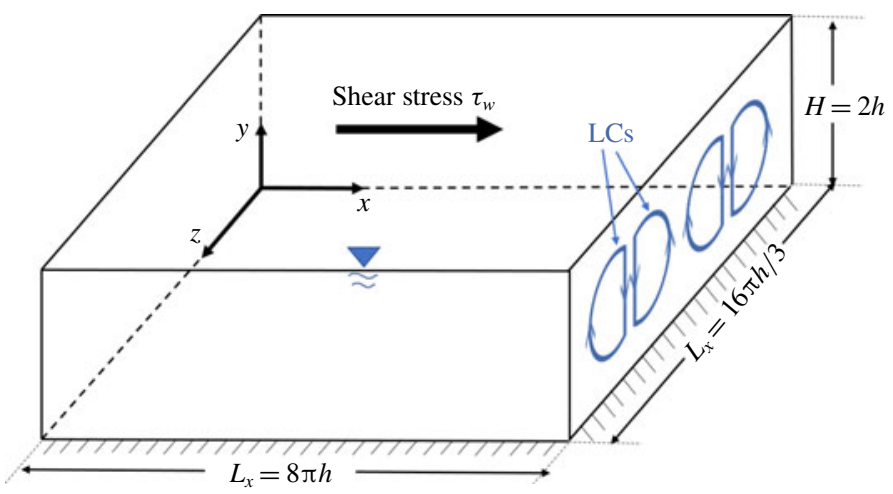

FIgURE 1. (Colour online) Sketch of the computational model for shear-driven shallowwater turbulence with LCs.

LES at higher Reynolds numbers with a larger computational domain size. In the present study, we perform wall-resolved LES of shallow-water Langmuir turbulence at $R e_{\tau}=395,700$ and 1000 to study the Reynolds number effect. The effects of dimensionless parameters $k h$ and $L a_{t}$ are also investigated.

The remainder of this paper is organized as follows. In $\S 2$, the governing equations and numerical method are introduced. In $\S 3$, the reappearance of the logarithmic layer at $R e_{\tau}=1000$ is reported, and the effects of $k h$ and $L a_{t}$ on the width of the logarithmic layer are studied. In $\S 4$, the Reynolds stresses are analysed. The reappearance of the logarithmic layer at $R e_{\tau}=1000$ is explained by analysing the Reynolds shear stress. The bimodal shape in the profile of the variance of streamwise velocity fluctuations at $R e_{\tau}=1000$ is reported and analysed based on the decomposition of the velocity fluctuations into a LC-coherent part and a residual turbulence part. In $\S 5$, the budget of turbulence kinematic energy (TKE) is analysed to further study the effects of LCs on the turbulence statistics, followed by the summary in $\S 6$.

\section{Simulation set-up and numerical method}

\subsection{Governing equations}

Figure 1 shows the computational domain and coordinates used in the present simulations. As shown, $x, y$ and $z$ represent the streamwise, vertical and spanwise directions, respectively. The water depth is $H$. Wall-resolved LES based on the $\mathrm{C}-\mathrm{L}$ equations is performed to study the neutrally stratified shallow-water Langmuir turbulence. Following McWilliams et al. (1997), a low-pass spatial filter is applied to obtain the wave-phase-averaged motions. The $\mathrm{C}-\mathrm{L}$ equations read

$$
\begin{gathered}
\nabla \cdot \overline{\boldsymbol{u}}=0, \\
\frac{\partial \overline{\boldsymbol{u}}}{\partial t}+\overline{\boldsymbol{u}} \cdot \nabla \overline{\boldsymbol{u}}=-\nabla \bar{\Pi}+v \nabla^{2} \overline{\boldsymbol{u}}+\nabla \cdot \boldsymbol{\tau}^{s g s}+\boldsymbol{u}_{s} \times \overline{\boldsymbol{\omega}} .
\end{gathered}
$$

Here, $\overline{\boldsymbol{u}}=(\bar{u}, \bar{v}, \bar{w})$ is the resolved velocity with $\bar{u}, \bar{v}$ and $\bar{w}$ denoting the velocity in the $x$-, $y$ - and $z$-directions, respectively. The overline represents the implicit grid-level filter. The effective pressure $\bar{\Pi}=\bar{p} / \rho+\bar{\Gamma}$ includes the resolved pressure $\bar{p} / \rho$ and the additional term (McWilliams et al. 1997)

$$
\bar{\Gamma}=\frac{1}{2} \boldsymbol{u}_{s} \cdot \boldsymbol{u}_{s}+\boldsymbol{u}_{s} \cdot \overline{\boldsymbol{u}} .
$$


In (2.2) and (2.3), $\boldsymbol{u}_{s}$ is the Stokes drift velocity induced by water waves. According to Longuet-Higgins (1953) and Phillips (1967), $\boldsymbol{u}_{s}$ induced by shallow-water potential waves is given as

$$
\boldsymbol{u}_{s}(y)=u_{s}^{*}\left(\frac{\cosh (2 k y)}{2 \sinh ^{2}(2 k h)}, 0,0\right)=\frac{u_{\tau}}{L a_{t}^{2}}\left(\frac{\cosh (2 k y)}{2 \sinh ^{2}(2 k h)}, 0,0\right) .
$$

Here, $u_{s}^{*}=\sigma k a^{2}$ is the characteristic value of $u_{s}$ (Tejada-Martínez \& Grosch 2007), with $\sigma, k$ and $a$ being the frequency, wavenumber and wave amplitude, respectively. In (2.2), $v$ is the kinematic viscosity, and the subgrid-scale (SGS) stress tensor $\boldsymbol{\tau}^{s g s}$ is included due to the use of the low-pass spatial filter. The last term on the right-hand side of (2.2) is the $\mathrm{C}-\mathrm{L}$ vortex force, with $\overline{\boldsymbol{\omega}}=\boldsymbol{\nabla} \times \overline{\boldsymbol{u}}$ being the vorticity. The value of $L a_{t}^{2}$ characterizes the relative magnitude of the wind-shear forcing with respect to wave forcing. According to the definitions of the C-L vortex force (2.2) and Stokes drift (2.4), the increase of either $L a_{t}$ or $k h$ leads to the magnitude decrease of the $\mathrm{C}-\mathrm{L}$ vortex force. Particularly, as the value of $L a_{t}$ approaches infinity, the flow reduces to a shear-driven turbulence without LCs.

In the C-L equations derived by Craik \& Leibovich (1976) and Leibovich (1977), the Stokes drift $u_{s}$ is obtained using the leading-order solution of potential waves with respect to the wave steepness $\epsilon$. Near the wave surface and water bottom, the magnitude of Stokes drift in the context of viscous flow is different from that obtained from the potential theory due to the viscous boundary condition. For example, at the water bottom, the Stokes drift $u_{s}$ is zero due to the no-slip boundary condition, but the value given by (2.4) is non-zero. Nevertheless, the magnitude of the latter is very small. Among all the cases studied in this paper, the maximal value of $u_{s}$ is $2.26 u_{\tau}$. Near the water surface, the viscous effect can also influence the Stokes drift. However, the influence of the viscous effect on the Stokes drift is confined in a thin layer. Longuet-Higgins (1953) showed that the leading-order approximation of shallow-water waves satisfies the potential solution in most of the water column, and only deviates from the potential solution in a thin viscous layer with a thickness comparable to that of the Stokes layer. The thickness of the Stokes layer $\delta_{s}$ is $\sqrt{2 v / \sigma}$, which is small for water waves. For example, in the case of finite-depth waves, if $\sigma=2 \pi \mathrm{rad} \mathrm{s}^{-1}$, the thickness of its Stokes layer is only $0.56 \mathrm{~mm}$ (Longuet-Higgins 1953). The thickness of the Stokes layer can also be estimated from other studies of turbulent boundary layer subjected to an oscillatory pressure gradient. Based on the results of Ramaprian \& Tu (1983), Scotti \& Piomelli (2001) and Manna, Vacca \& Verzicco (2012), we can estimate that $\delta_{s}$ is smaller than five wall units under the wave condition that Langmuir circulations are observed in the field by Gargett \& Wells (2007). This above discussions indicate that the viscous effects are confined in the viscous sublayer. According to the original theory of Craik \& Leibovich (1976), the viscous effects may lead to a correction to the $\mathrm{C}-\mathrm{L}$ equation. However, the correction is only subtle and is in a thin layer near the wave surface and water bottom. In Langmuir turbulence, due to the wind shear, the viscous effects near the wave surface and water bottom are much less important than the wind-induced shear. As a result of the above factors, it is believed that the viscous effects at the boundaries only have higher-order influence on the dynamics of the Langmuir turbulence. It was shown in previous LES studies of shallow-water Langmuir turbulence that the LES results based on the C-L equations with $u_{s}$ given by (2.4) (Tejada-Martínez \& Grosch 2007) agree well with the field measurement data (Gargett \& Wells 2007). At last, we remark that when more computer power becomes available, one might conduct wave-phaseresolved simulations with full viscous boundary conditions to quantify the viscous 


\begin{tabular}{lcccccccccccc}
\hline Case & $R e_{\tau}$ & $L a_{t}$ & $k h$ & $\lambda / H$ & $L_{x} / h$ & $L_{z} / h$ & $N_{x}$ & $N_{y}$ & $N_{z}$ & $\Delta x^{+}$ & $\Delta y_{\min }^{+}$ & $\Delta z^{+}$ \\
Case 1 & 1000 & 0.7 & 0.5 & $2 \pi$ & $8 \pi$ & $16 \pi / 3$ & 512 & 192 & 512 & 49.09 & 0.625 & 32.72 \\
Case 2 & 700 & 0.7 & 0.5 & $2 \pi$ & $8 \pi$ & $16 \pi / 3$ & 384 & 144 & 384 & 45.81 & 0.588 & 30.54 \\
Case 3S & 395 & 0.7 & 0.5 & $2 \pi$ & $4 \pi$ & $8 \pi / 3$ & 128 & 128 & 128 & 38.78 & 0.395 & 25.85 \\
Case 3 & 395 & 0.7 & 0.5 & $2 \pi$ & $8 \pi$ & $16 \pi / 3$ & 256 & 128 & 256 & 38.78 & 0.395 & 25.85 \\
Case 3L & 395 & 0.7 & 0.5 & $2 \pi$ & $32 \pi$ & $64 \pi / 3$ & 1024 & 128 & 1024 & 38.78 & 0.395 & 25.85 \\
Case 4 & 1000 & 0.7 & 1.0 & $\pi$ & $8 \pi$ & $16 \pi / 3$ & 512 & 192 & 512 & 49.09 & 0.625 & 32.72 \\
Case 5 & 1000 & 0.7 & 1.5 & $2 \pi / 3$ & $8 \pi$ & $16 \pi / 3$ & 512 & 192 & 512 & 49.09 & 0.625 & 32.72 \\
Case 6 & 1000 & 0.7 & 2.0 & $\pi / 2$ & $8 \pi$ & $16 \pi / 3$ & 512 & 192 & 512 & 49.09 & 0.625 & 32.72 \\
Case 7 & 1000 & 0.4 & 0.5 & $2 \pi$ & $8 \pi$ & $16 \pi / 3$ & 512 & 192 & 512 & 49.09 & 0.625 & 32.72 \\
Case 8 & 1000 & 0.9 & 0.5 & $2 \pi$ & $8 \pi$ & $16 \pi / 3$ & 512 & 192 & 512 & 49.09 & 0.625 & 32.72 \\
Case 9 & 1000 & $\infty$ & - & - & $8 \pi$ & $16 \pi / 3$ & 512 & 192 & 512 & 49.09 & 0.625 & 32.72
\end{tabular}

TABLE 1. Key parameters of simulation cases.

effects at the boundaries, which requires a simulation framework different from the present one based on the $\mathrm{C}-\mathrm{L}$ equations and is beyond the scope of this work.

The surface boundary for the wave-phase-averaged motions described in the $\mathrm{C}-\mathrm{L}$ equations is a rigid lid, which is obtained by keeping the leading-order term in the expansion of the boundary condition with respect to the wave steepness (Craik \& Leibovich 1976; Leibovich 1977). According to Kemp \& Simons (1982) and McWilliams, Restrepo \& Lane (2004), the difference in wave amplitude induced by wave-current interactions is a higher-order effect with respect to the wave steepness, which is omitted in the $\mathrm{C}-\mathrm{L}$ theory. Therefore, it is believed that the $\mathrm{C}-\mathrm{L}$ equation with a rigid-lid boundary condition at wave surface can still be used under the influence of wave-current interaction. In fact, the rigid-lid surface boundary condition has been widely used in LES of Langmuir turbulence (Skyllingstad \& Denbo 1995; McWilliams et al. 1997; Tejada-Martínez \& Grosch 2007). The flow is driven by a constant wind-shear stress $\tau_{w}=\rho u_{\tau}^{2}$ at the water surface $y=2 h$, with $\rho$ being the density of water. The no-slip boundary condition is applied at the bottom $y=0$. Periodic boundary conditions are imposed in the $x$ - and $z$-directions.

\subsection{Computational parameters}

Table 1 summarizes the key parameters of the present simulations. In the table, $L_{x}$ and $L_{z}$ denote the computation domain size in the $x$ - and $z$-directions, respectively; $N_{x}$, $N_{y}$ and $N_{z}$ are the number of the grid points in the $x$-, $y$ - and $z$-directions, respectively, with $\Delta x^{+}, \Delta y_{\min }^{+}$and $\Delta z^{+}$being the grid resolution in the corresponding directions. The superscript ' + ' denotes flow quantities normalized using the viscous length scale $v / u_{\tau}$ and surface friction velocity $u_{\tau}$. Note that due to the use of a conservative finite difference scheme for the discretization in the $y$-direction (Kim \& Moin 1985), the shear stress $\tau_{w}$ prescribed at the top is balanced by the time-averaged shear stress at the bottom when the flow is fully developed to a statistically stationary state. Correspondingly, the value of $u_{\tau}$ at the water bottom obtained from the simulation is also identical to that at the water surface. As a result, the values of $\Delta x^{+}, \Delta y_{\min }^{+}$ and $\Delta z^{+}$are all constants in different cases with the same $R e_{\tau}$. The details of the numerical scheme are given in $\S 2.3$.

Simulation cases 1-3 are conducted to study the effect of the Reynolds number $R e_{\tau}=u_{\tau} h / v$. The value of $R e_{\tau}$ is 1000, 700 and 395 in cases 1, 2 and 3, respectively. 
The dimensionless parameters $L a_{t}=0.7$ and $k h=0.5$ are chosen to match the typical field condition in the observation of Gargett \& Wells (2007). Cases 3S and 3L are performed to examine the influence of the computational domain size on the simulation results. The values of $R e_{\tau}, L a_{t}$ and $k h$ in cases $3 \mathrm{~S}$ and $3 \mathrm{~L}$ are the same as those in case 3 . The computational domain sizes of cases $3 \mathrm{~S}$ and $3 \mathrm{~L}$ are respectively one-half and four times that of case 3 in both the $x$ - and $z$-directions. It is shown in $\$ 2.4$ that the computation domain size of case $3, L_{x} \times L_{z}=8 \pi h \times 16 \pi h / 3$, is sufficiently large to obtain accurate simulation results. As a result, the computational domain size of the other cases is set to that of case 3. Cases 1 and 4-6 are performed to investigate the effect of $k h$. In these cases, the value of $k h$ varies from 0.5 to 2.0 with the corresponding wavelength-to-depth ratio $\lambda / H$ varying from $2 \pi$ to $\pi / 2$, while $R e_{\tau}$ and $L a_{t}$ are fixed at 1000 and 0.7 , respectively. The effect of $L a_{t}$ is studied through cases 1,7 and 8 . The value of $L a_{t}$ is $0.4,0.7$ and 0.9 in cases 7,1 and 8, respectively. Case 9 is the pure shear-driven turbulence case, which is used as a reference case to reveal the influence of LCs on turbulence statistics.

The grid is evenly distributed in the $x$ - and $z$-directions. In the $y$-direction, the grid lines are located at $y_{j} / h=b^{-1} \tanh \left[\left(-1+2 j / N_{y}\right) \tanh ^{-1}(b)\right]+1$ for $j=0,1, \ldots, N_{y}$ with $b=0.973$, such that the grid is clustered near the water surface and water bottom. As shown in table 1 , the minimum vertical grid resolution $\Delta y_{\min }^{+}$near the top and bottom is smaller than 1.0, satisfying the grid resolution of wall-resolved LES. The grid resolution in the $x$ - and $z$-directions also satisfies the standard of wall-resolved LES, i.e. $\Delta x^{+} \simeq 50$ and $\Delta z^{+} \simeq 30$ (Chapman 1979; Choi \& Moin 2012). We have re-run case 1 by doubling the number of grid points in the $x$ - and $z$-directions to examine the effect of grid resolution on the simulation results. It is found that the mean velocity and Reynolds stresses change by less than $2 \%$ and $4 \%$, respectively.

\subsection{Numerical method}

In our simulations, the fractional-step projection method (Kim \& Moin 1985) is employed to solve (2.1) and (2.2). The second-order explicit Adams-Bashforth scheme and second-order implicit Crank-Nicolson scheme are used for the time integrations of the convective and viscous terms in the $\mathrm{C}-\mathrm{L}$ equations, respectively. In the $x$ - and $z$-directions, the Fourier-Galerkin method is utilized for the spatial discretization. The $3 / 2$ rule is employed to remove the aliasing error. In the vertical direction, the second-order finite-difference method is used to discretize the flow field. The vertical velocity component $\bar{v}$ is defined at grid points, while horizontal velocity components $\bar{u}, \bar{w}$, and pressure $\bar{\Pi}$ are defined at the centres of grid lines.

The SGS stress $\tau_{i j}^{\text {sgs }}$ is calculated using the dynamic Smagorinsky model (Germano et al. 1991; Lilly 1992). Hereinafter, the subscript $i$ (or $j)=1,2$ and 3 represents the component in the streamwise, vertical and spanwise directions, respectively. The SGS stress $\tau_{i j}^{s g s}$ is calculated as

$$
\tau_{i j}^{s g s}=\frac{1}{3} \tau_{k k}^{s g s} \delta_{i j}+2 C_{s} \bar{\Delta}^{2}|\bar{S}| \bar{S}_{i j},
$$

where the dilatational part of the SGS stress $\tau_{k k}^{s g s} \delta_{i j} / 3$ is absorbed into the modified pressure $\bar{\Pi}, C_{s}$ is the model coefficient, $\bar{\Delta}=(\Delta x \Delta y \Delta z)^{1 / 3}$ is the width of the gridlevel filter, $\bar{S}_{i j}=\left(\partial \bar{u}_{i} / \partial x_{j}+\partial \bar{u}_{j} / \partial x_{i}\right) / 2$ is the resolved strain-rate tensor and $|\bar{S}|=$ $\left(2 \bar{S}_{i j} \bar{S}_{i j}\right)^{1 / 2}$ is the norm of $\bar{S}_{i j}$. The model coefficient $C_{s}$ is determined using a dynamic procedure as

$$
C_{s}=\frac{1}{2} \frac{\left\langle L_{i j} M_{i j}\right\rangle_{x z}}{\left\langle M_{i j} M_{i j}\right\rangle_{x z}},
$$



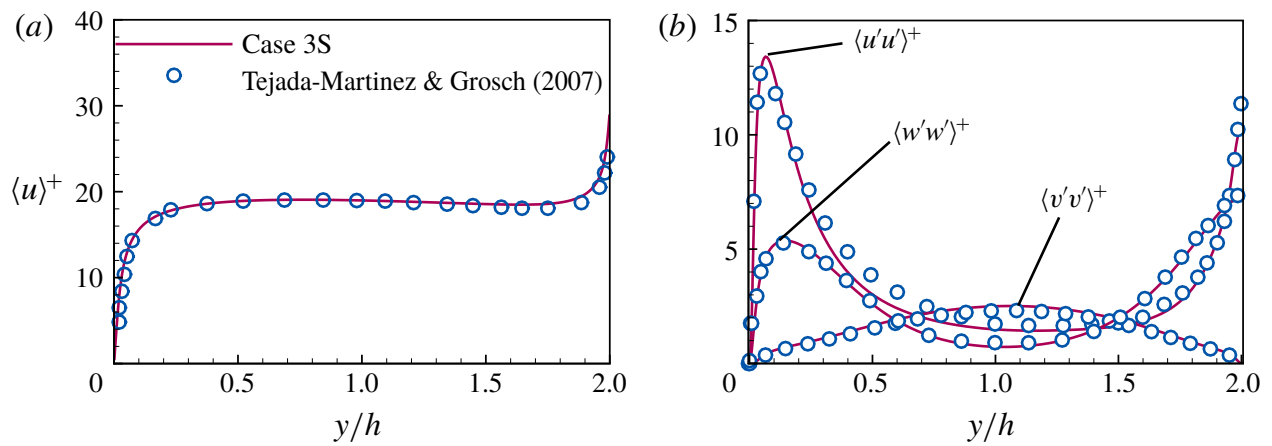

Figure 2. (Colour online) Profiles of $(a)$ the mean streamwise velocity $\langle u\rangle^{+}$and $(b)$ Reynolds normal stresses in case $3 \mathrm{~S}$ and comparison with the result of Tejada-Martínez \& Grosch (2007).

where

$$
L_{i j}=\widetilde{\bar{u}}_{i} \widetilde{\bar{u}}_{j}-\widetilde{\bar{u}_{i} \bar{u}_{j}}
$$

and

$$
M_{i j}=\widetilde{\Delta}^{2} \mid \widetilde{\bar{S}}_{\mid \bar{S}_{i j}}-\widetilde{\bar{\Delta}^{2}|\bar{S}| \bar{S}_{i j}}
$$

Here, \langle\rangle$_{x z}$ denotes the averaging in the $x$ - and $z$-directions, the tilde stands for a testgrid-level filter, and $\widetilde{\Delta}$ is the size of the test-grid-level filter.

To validate the code, we compare the results in case $3 \mathrm{~S}$ with those in TejadaMartínez \& Grosch (2007). The computational domain size, $L a_{t}$, and $R e_{\tau}$ in case $3 \mathrm{~S}$ are the same as those in Tejada-Martínez \& Grosch (2007), while the value of $k h$ in case $3 \mathrm{~S}(0.5)$ is close to that (0.52) in Tejada-Martínez \& Grosch (2007). Figure 2 compares the profiles of the mean streamwise velocity $\langle u\rangle^{+}$and Reynolds normal stresses $\left\langle u^{\prime} u^{\prime}\right\rangle^{+},\left\langle v^{\prime} v^{\prime}\right\rangle^{+}$and $\left\langle w^{\prime} w^{\prime}\right\rangle^{+}$in case $3 \mathrm{~S}$ with those in Tejada-Martínez \& Grosch (2007) to validate the numerical algorithms. Here, the angular brackets \langle\rangle represent the temporal and spatial averaging over a time duration of $t=120 \mathrm{~h} / u_{\tau}$ in $x-z$ planes. The prime denotes fluctuations. For the convenience of presenting the results, the overline is omitted hereinafter. Figures $2(a)$ and $2(b)$ show that the results of case $3 \mathrm{~S}$ are close to those of Tejada-Martínez \& Grosch (2007). In the equilibrium shallow-water Langmuir turbulence, the viscous shear stress $\mathrm{d}\langle u\rangle^{+} / \mathrm{d} y^{+}$, resolved Reynolds shear stress $-\left\langle u^{\prime} v^{\prime}\right\rangle^{+}$and the SGS shear stress $\left\langle\tau_{12}^{s g s}\right\rangle^{+}$ satisfy (Tejada-Martínez \& Grosch 2007)

$$
\mathrm{d}\langle u\rangle^{+} / \mathrm{d} y^{+}-\left\langle u^{\prime} v^{\prime}\right\rangle^{+}+\left\langle\tau_{12}^{s g s}\right\rangle^{+}=1 .
$$

Figure 3 shows different terms in (2.9) in Langmuir turbulence (case 3S) and shear-driven turbulence (case 1). As shown, the viscous stress is important near the water bottom, while the resolved Reynolds shear stress is dominant away from the bottom of the water. The SGS shear stress is significantly smaller than the resolved Reynolds shear stress. The value of the total shear stress obtained from our LES is 1.0 throughout the water column, indicating that the simulation reaches a statistically equilibrium state and the time duration used for performing time averaging is sufficiently long. 
(a)

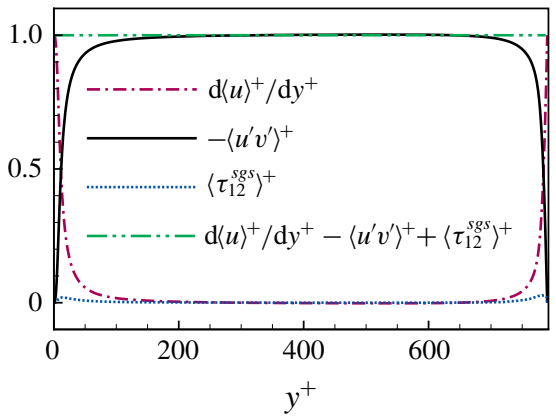

(b)

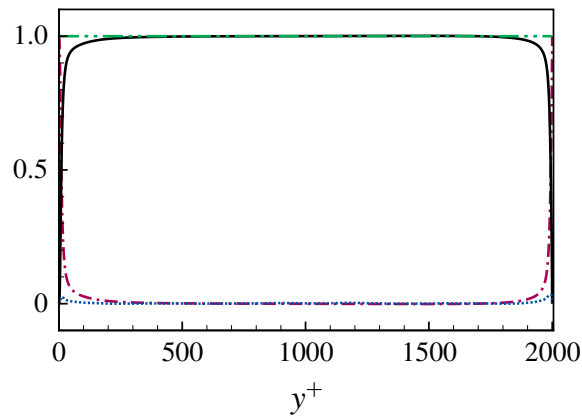

Figure 3. (Colour online) Profiles of shear stresses in $(a)$ case $3 \mathrm{~S}$ and $(b)$ case 1.

(a)
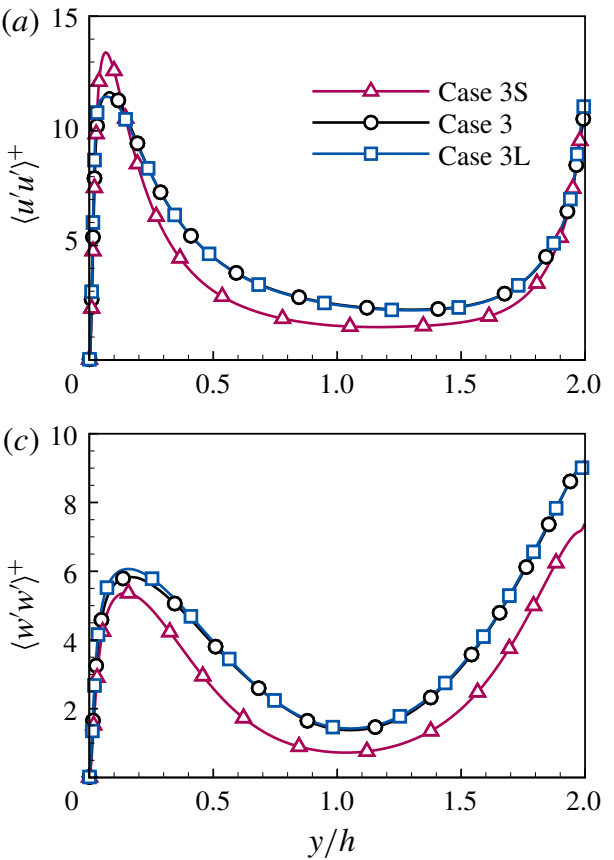

(b)

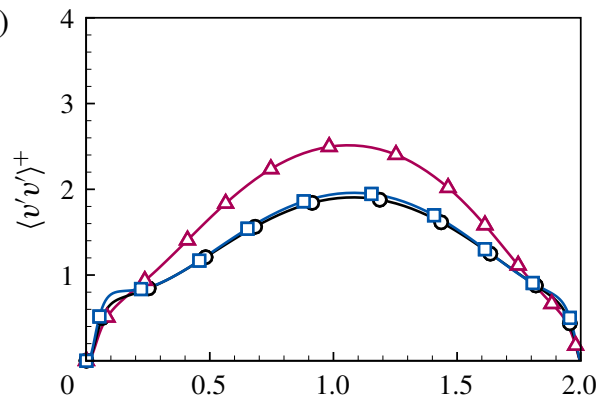

(d)

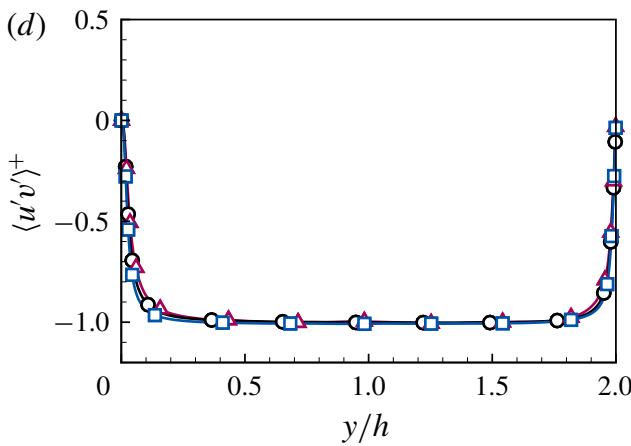

FIgURE 4. (Colour online) Profiles of $(a)\left\langle u^{\prime} u^{\prime}\right\rangle^{+},(b)\left\langle v^{\prime} v^{\prime}\right\rangle^{+},(c)\left\langle w^{\prime} w^{\prime}\right\rangle^{+}$and $(d)\left\langle u^{\prime} v^{\prime}\right\rangle^{+}$ in cases $3 \mathrm{~S}, 3$ and $3 \mathrm{~L}$ with different domain sizes.

\subsection{Effect of computational domain size}

Figure 4 compares the profiles of $\left\langle u^{\prime} u^{\prime}\right\rangle^{+},\left\langle v^{\prime} v^{\prime}\right\rangle^{+},\left\langle w^{\prime} w^{\prime}\right\rangle^{+}$and $\left\langle u^{\prime} v^{\prime}\right\rangle^{+}$among cases 3S, 3 and 3L to examine the effect of computational domain size on the Reynolds stresses. As shown, the profiles of $\left\langle u^{\prime} u^{\prime}\right\rangle^{+},\left\langle v^{\prime} v^{\prime}\right\rangle^{+},\left\langle w^{\prime} w^{\prime}\right\rangle^{+}$and $\left\langle u^{\prime} v^{\prime}\right\rangle^{+}$in case 3 collapse with those in case $3 \mathrm{~L}$, indicating that the computational domain size of case 3 is adequate to obtain domain-size-independent results of Reynolds stresses. The domain size of case $3 \mathrm{~S}$, on the other hand, is inadequate as evident by the difference in the profiles of $\left\langle u^{\prime} u^{\prime}\right\rangle^{+},\left\langle v^{\prime} v^{\prime}\right\rangle^{+}$and $\left\langle w^{\prime} w^{\prime}\right\rangle^{+}$of case 3S from those of cases 3 and 3L (figure $4 a-c$ ).

We have also examined the effect of computational domain size on the scales and intensities of LCs. The LC quantities are extracted using a triple decomposition, 

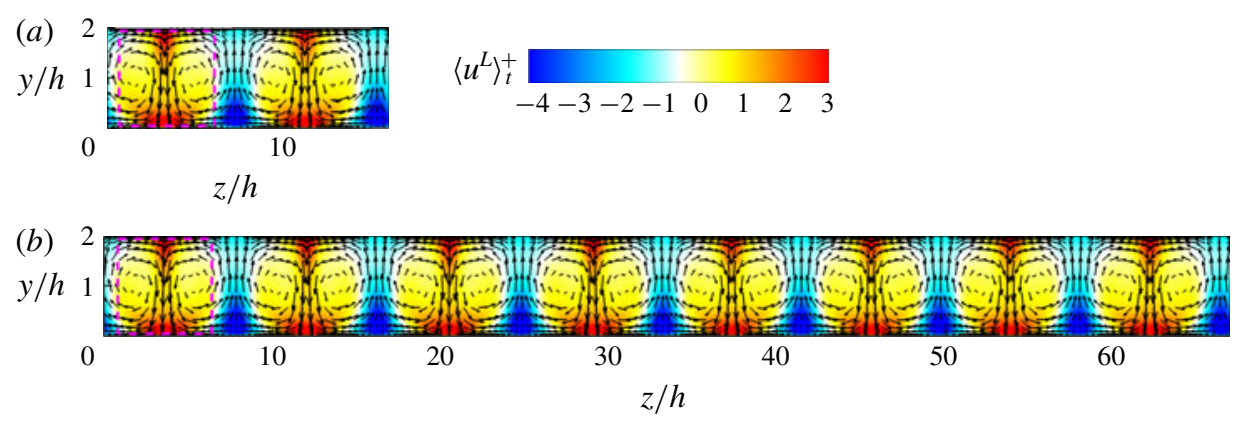

Figure 5. (Colour online) Contours of $\left\langle u^{L}\right\rangle_{t}^{+}$and vectors consisting of $\left(\left\langle w^{L}\right\rangle_{t}^{+},\left\langle v^{L}\right\rangle_{t}^{+}\right)$in (a) case 3 and (b) case $3 \mathrm{~L}$ with different domain sizes.

viz.

$$
\boldsymbol{u}=\langle\boldsymbol{u}\rangle+\boldsymbol{u}^{\prime}=\langle\boldsymbol{u}\rangle+\boldsymbol{u}^{L}+\boldsymbol{u}^{T}
$$

Because the LCs are elongated along the wind direction (Gargett et al. 2004; Gargett $\&$ Wells 2007), the LC-coherent velocity $\boldsymbol{u}^{L}$ is defined as the streamwise averaging of $\boldsymbol{u}^{\prime}$. The residual turbulence fluctuations are denoted as $\boldsymbol{u}^{T}$. The summation of $\boldsymbol{u}^{L}$ and $\boldsymbol{u}^{T}$ gives the total fluctuation $\boldsymbol{u}^{\prime}$. The same triple decomposition method was used by Tejada-Martínez \& Grosch (2007), Kukulka et al. (2012) and Tejada-Martínez et al. (2013) in shallow-water Langmuir turbulence.

Figure 5 compares the LCs obtained from cases 3 and 3L. The LCs are visualized using the vectors consisting of $\left(\left\langle w^{L}\right\rangle_{t}^{+},\left\langle v^{L}\right\rangle_{t}^{+}\right)$and the contours of $\left\langle u^{L}\right\rangle_{t}^{+}$in an $z-y$ plane, where \langle\rangle$_{t}$ represents the time averaging over $120 h / u_{\tau}$. As highlighted by the red dashed boxes in figure $5(a, b)$, the positive $u^{L}$ forms a mushroom-shaped region (Tejada-Martínez \& Grosch 2007), and each mushroom is accompanied by a pair of counter-rotating streamwise vortices, consistent with the observation of Tejada-Martínez \& Grosch (2007). As pointed out by Tejada-Martínez et al. (2012) and Sinha et al. (2015), the magnitude of the time- and streamwise-averaged velocity is larger in the downwelling limbs than in the upwelling limbs, because $u^{L}$ is positive and negative in the downwelling and upwelling limbs, respectively (figure 5). Furthermore, in the region away from the bottom, the decrease in the amplitude of $u^{L}$ with $y / h$ (figure 5) results in a negative and positive vertical gradient of $u^{L}$ in the downwelling and upwelling limbs, such that the velocity gradient in the upwelling limbs is relatively small in comparison with that in the downwelling limbs. Two and eight pairs of counter-rotating vortices are observed in cases 3 and 3L, respectively. The spanwise length scale of LCs obtained from the present LES is $8 \pi h / 3$, agreeing with that in Tejada-Martínez \& Grosch (2007). The spanwise domain size in the present study follows Tejada-Martínez \& Grosch (2007), who set the spanwise domain size to $8 \pi h / 3$, which approximates the spanwise length scale of LCs in the field measurements by Gargett et al. (2004) and Gargett \& Wells (2007). According to Tejada-Martínez et al. (2012), no Langmuir circulation is observed if the spanwise domain size is set to $12 \pi h / 3$.

The Reynolds stress can be further decomposed into the LC-coherent part and residual turbulence part as

$$
\left\langle u_{i}^{\prime} u_{j}^{\prime}\right\rangle=\left\langle u_{i}^{L} u_{j}^{L}\right\rangle+\left\langle u_{i}^{T} u_{j}^{T}\right\rangle
$$




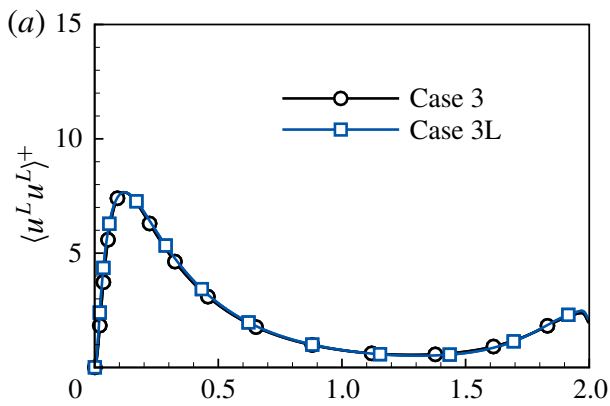

(b)
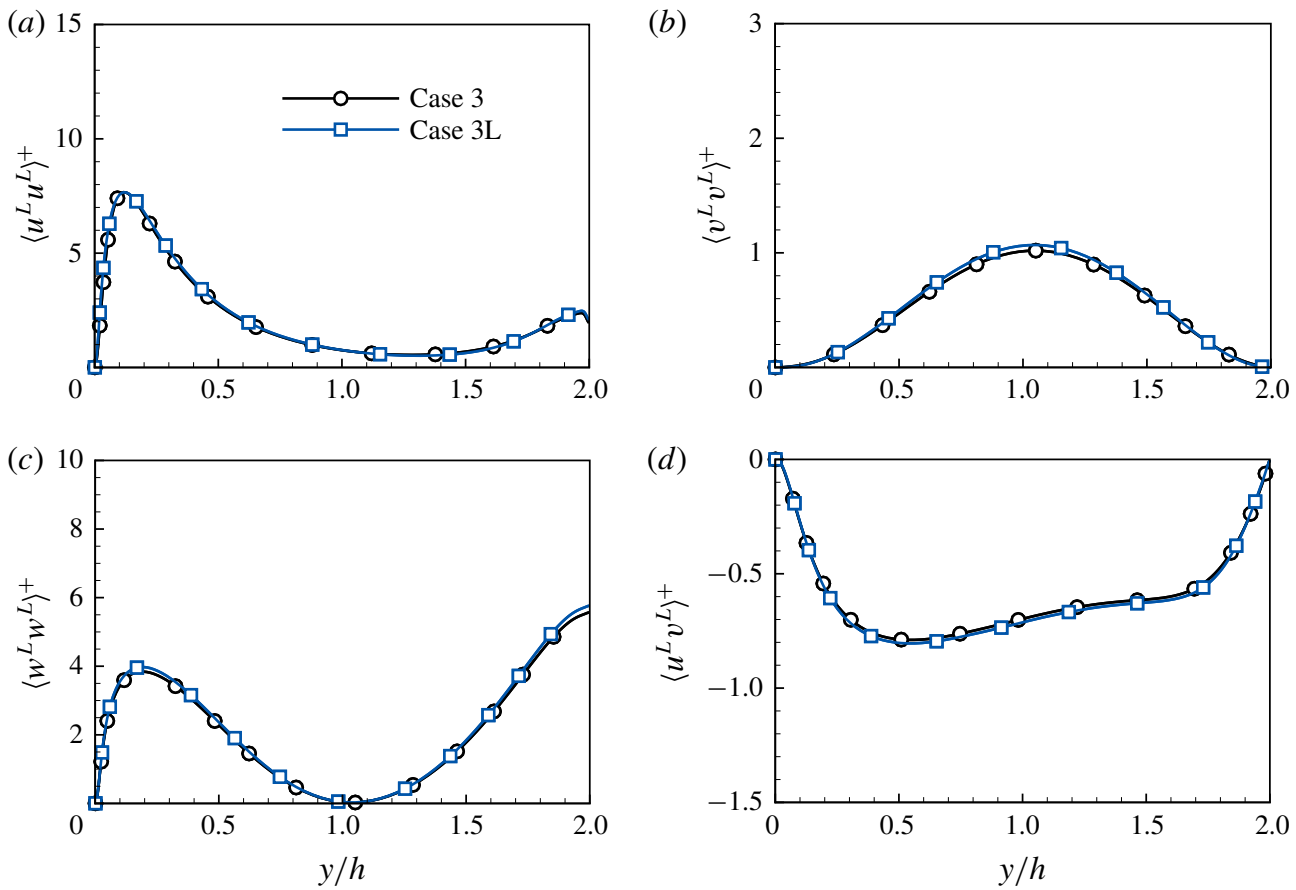

Figure 6. (Colour online) Profiles of $(a)\left\langle u^{L} u^{L}\right\rangle^{+},(b)\left\langle v^{L} v^{L}\right\rangle^{+},(c)\left\langle w^{L} w^{L}\right\rangle^{+}$and $(d)$ $\left\langle u^{L} v^{L}\right\rangle^{+}$in cases 3 and $3 \mathrm{~L}$ with different domain sizes.

The cross-term $\left\langle u_{i}^{L} u_{j}^{T}\right\rangle$ is zero (Akan et al. 2013; Martinat et al. 2014), because $u_{i}^{L}$ are streamwise averaged and the streamwise-averaged value of $u_{j}^{T}$ is zero. To further examine the domain-size effect on the intensity of LCs, we compare the profiles of LC-induced Reynolds stresses $\left\langle u^{L} u^{L}\right\rangle^{+},\left\langle v^{L} v^{L}\right\rangle^{+},\left\langle w^{L} w^{L}\right\rangle^{+}$and $\left\langle u^{L} v^{L}\right\rangle^{+}$between cases 3 and $3 \mathrm{~L}$ in figure 6 . As shown, the profiles in case 3 agree with those in case $3 \mathrm{~L}$. The results shown in figures 4-6 indicate that the computational domain size of $L_{x} \times L_{z}=$ $8 \pi h \times 16 \pi h / 3$ is sufficiently large for capturing LCs.

\section{Mean velocity}

It was reported in Tejada-Martínez et al. (2012) that the logarithmic layer over the bottom boundary is disrupted in the shallow-water Langmuir turbulence at $R e_{\tau}=395$ and 590 with $L a_{t}=0.7$ and $k h=0.5$. In this paper, we start our analysis from the examination of the logarithmic law of the mean velocity at higher Reynolds numbers. In the logarithmic layer, the mean velocity satisfies (Millikan 1938; Rotta 1962; Townsend 1976)

$$
\langle u\rangle^{+}=\frac{1}{\kappa} \log \left(y^{+}\right)+B,
$$

where $\kappa$ is the von Kármán constant, and $B$ is the intercept. The logarithmic law can be quantitatively identified by the diagnostic function

$$
F=y^{+} \frac{\mathrm{d}\langle u\rangle^{+}}{\mathrm{d} y^{+}} .
$$



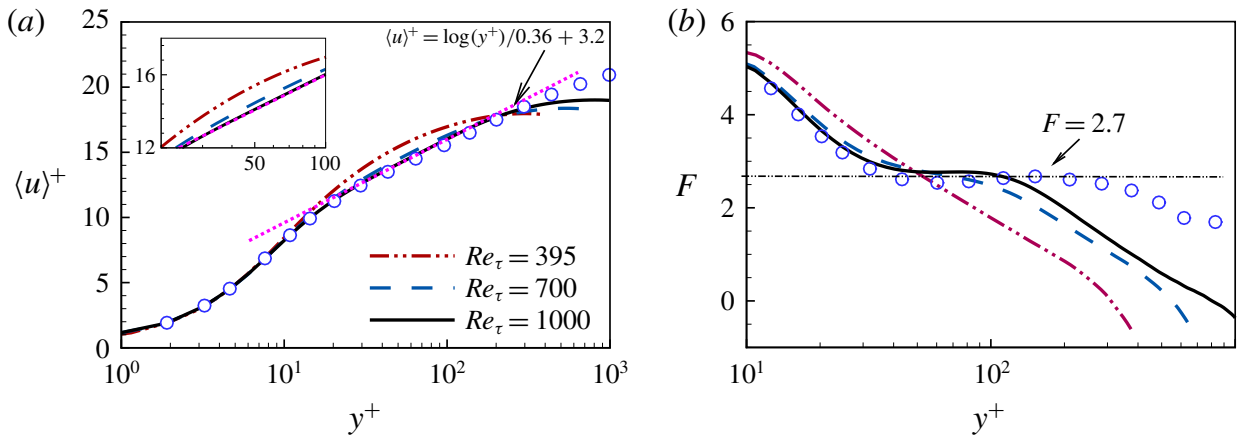

FIgURE 7. (Colour online) Effect of Reynolds number on $(a)\langle u\rangle^{+}$and $(b) F$ in the bottom half of the shallow-water Langmuir turbulence in cases $1-3$ with $R e_{\tau}=1000,700$ and 395, respectively. The logarithmic law in $(a)$ is $\langle u\rangle^{+}=\log \left(y^{+}\right) / 0.36+3.2$. The open circles represent the results of case 9 (the shear-driven turbulence at $R e_{\tau}=1000$ ).

The value of $F$ is a constant $1 / \kappa$ if the logarithmic law (3.1) holds. However, at a finite Reynolds number, the value of $F$ is not strictly identical to $1 / \kappa$, but increases slightly with $y^{+}$in a quasi-plateau region (Moser, Kim \& Mansour 1999; Hoyas \& Jiménez 2006; Avsarkisov et al. 2014; Bernardini, Pirozzoli \& Orlandi 2014; Lee \& Moser 2015). This is because the logarithmic law is only the first-order truncation of the asymptotic expansion of the mean velocity with respect to $1 / \operatorname{Re}_{\tau}$ (Afzal \& Yajnik 1973; Zagarola, Perry \& Smits 1997; Jiménez \& Moser 2007; Mizuno \& Jiménez 2011).

Figure 7 compares the profiles of $\langle u\rangle^{+}$and $F$ among cases 1, 2 and 3, with $R e_{\tau}=1000,700$ and 395, respectively. The results of case 9 for the shear-driven turbulence at $R e_{\tau}=1000$ are superimposed as a reference. As shown in figure 7(a), the profile of $\langle u\rangle^{+}$at $R e_{\tau}=395$ deviates from the logarithmic law, consistent with the results of Tejada-Martínez et al. (2012). However, the profile of $\langle u\rangle^{+}$ approaches the logarithmic law progressively as the Reynolds number increases. This observation from figure $7(a)$ is further confirmed by the diagnostic function $F$. Figure $7(b)$ shows that although the profile of $F$ in Langmuir turbulence deviates from that in shear-driven turbulence above $y^{+}=100$, the profile of $F$ below $y^{+}=100$ approaches that of shear-driven turbulence progressively as the Reynolds number increases. Particularly, at $R e_{\tau}=1000$, the profiles of $F$ in the Langmuir turbulence and shear-driven turbulence are close to each other below $y^{+}=100$, indicating a weak influence of LCs on the mean velocity in the near-bottom region. A plateau region can be observed in the profile of $F$ in the Langmuir turbulence at $R e_{\tau}=1000$, an indicator of the presence of logarithmic layer. The value of $F$ in the plateau region is approximately 2.77, and correspondingly, the value of the von Kármán constant is $\kappa=0.36$. Note that $\kappa$ is not necessarily the conventional value of 0.41 . It also deviates from 0.41 in canonical wall-bounded turbulence at finite Reynolds number (Nagib \& Chauhan 2008; Klewicki, Fife \& Wei 2009; Mizuno \& Jiménez 2011; Jiménez 2012; Marusic et al. 2013; Avsarkisov et al. 2014; Lee \& Moser 2015). The conventional value $\kappa=0.41$ is the asymptotic value as the Reynolds number approaches infinity (Jiménez \& Moser 2007; Mizuno \& Jiménez 2011). The presence of the logarithmic layer at $R e_{\tau}=1000$ indicates that the wall-layer model based on the logarithmic law is still appropriate in the shallow-water Langmuir turbulence at high Reynolds number. Because the logarithmic layer in Langmuir 

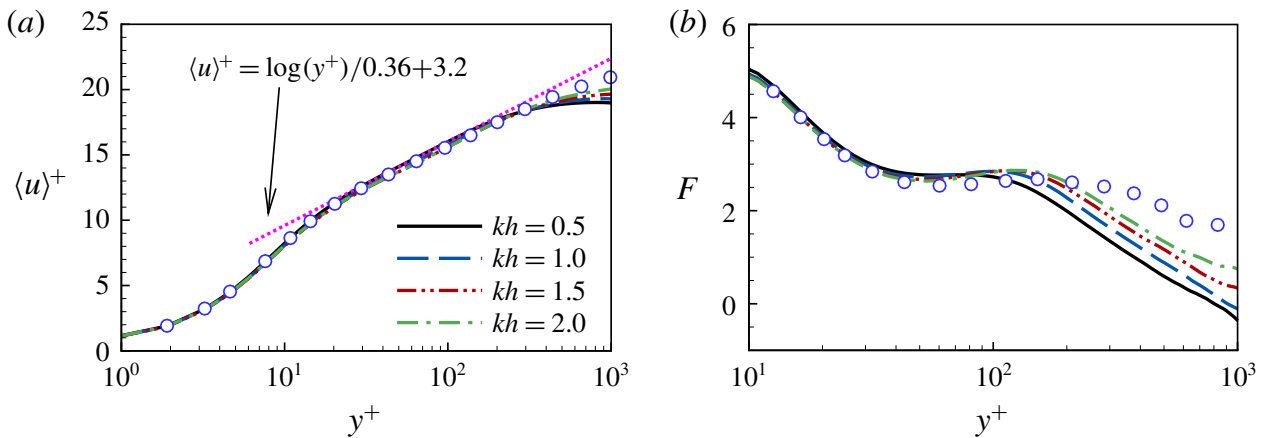

FIGURE 8. (Colour online) Effect of $k h$ on $(a)\langle u\rangle^{+}$and $(b)$ its diagnostic function $F$ in the bottom half of the shallow-water Langmuir turbulence in cases 1 and 4-6 with $k h=0.5,1,1.5$ and 2 , respectively. The open circles represent the results in case 9 (the shear-driven turbulence at $\left.R e_{\tau}=1000\right)$.
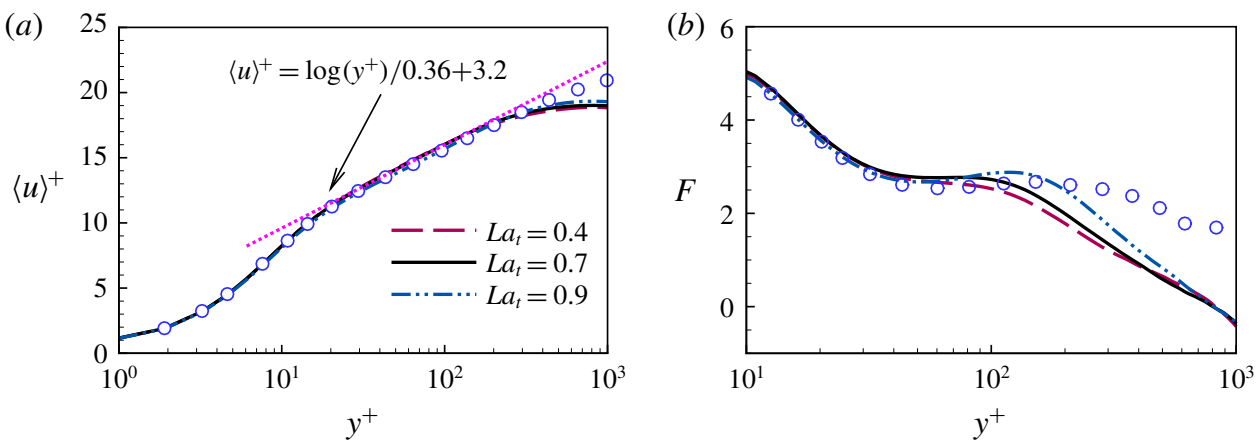

FIGURE 9. (Colour online) Effect of $L a_{t}$ on $(a)\langle u\rangle^{+}$and $(b)$ its diagnostic function $F$ in the bottom half of the flow in cases 1,7 and 8 with $L a_{t}=0.7,0.4$ and 0.9 , respectively. The open circles represent the results in case 9 (the shear-driven turbulence at $R e_{\tau}=1000$ ).

turbulence is thinner than that in shear-driven turbulence (figure $7 b$ ), care must be taken to ensure that the first grid is located in the logarithmic layer when the wall-layer model is used. The reappearance of the logarithmic layer at $R e_{\tau}=1000$ is further explained in $\S 4.2$, based on the analysis of Reynolds shear stress, an indicator of the transport of mean momentum in the vertical direction.

Figures 8 and 9 show respectively the effects of the dimensionless parameters $k h$ and $L a_{t}$ on the profiles of $\langle u\rangle^{+}$and $F$. The results in cases 1 and 4-6 with various values of $k h$ are shown in figure 8 , while those of cases 1,7 and 8 with various values of $L a_{t}$ are shown in figure 9 (see table 1 for the parameters). The results of case 9 for shear-driven turbulence are superimposed in figures 8 and 9 for comparison. As pointed out above, at $R e_{\tau}=1000$, the LCs do not significantly influence the mean velocity in the near-bottom region (figure 7). As a result, below $y^{+}=80$, both the profiles of $\langle u\rangle^{+}$and those of $F$ in cases 1 and 4-8 for Langmuir turbulence with various values of $k h$ and $L a_{t}$ are coincident with those in case 9 for shear-driven turbulence. As the value of either $k h$ or $L a_{t}$ increases, the magnitude of the vortex force decreases ((2.2) and (2.4)), resulting in a weaker influence of the vortex force on the mean velocity. Therefore, the profile of $F$ above $y^{+}=80$ in Langmuir turbulence 


$\begin{array}{lcccccc}\text { Case } & \text { Case } 1 & \text { Case } 4 & \text { Case 5 } & \text { Case 6 } & \text { Case } 8 & \text { Case } 9 \\ L a_{t} & 0.7 & 0.7 & 0.7 & 0.7 & 0.9 & \infty \\ k h & 0.5 & 1.0 & 1.5 & 2.0 & 0.5 & - \\ \kappa & 0.359 & 0.362 & 0.363 & 0.365 & 0.360 & 0.386\end{array}$

TABLE 2. The von Kármán constant $\kappa$ obtained at $R e_{\tau}=1000$.

approaches that in shear-driven turbulence as the values of $k h$ and $L a_{t}$ increase, leading to the thickness increase of the logarithmic layer (figures $8 b$ and $9 b$ ). The effect of $k h$ on the mean velocity at $R e_{\tau}=1000$ obtained from our LES is consistent with the trend observed by Tejada-Martínez et al. (2012) and Sinha et al. (2015) at $R e_{\tau}=$ 395. According to the analysis above, $R e_{\tau}, k h$ and $L a_{t}$ all influence the thickness of the logarithmic layer in shallow-water Langmuir turbulence. In the future, if more measurement and simulation data are available, it will be interesting to derive an explicit expression of the thickness of the logarithmic layer as a function of these three parameters, which is however, infeasible at this stage due to the limitation in the present computer power and beyond the scope of this study.

The von Kármán constant $\kappa$ is an important parameter in the wall-layer modelling of wall turbulence (Cabot \& Moin 2000; Piomelli \& Balaras 2002). Table 2 lists the values of $\kappa$ in different cases, calculated by fitting the mean velocity in the plateau region of $F$. Note that because the logarithmic layer is absent in cases 2,3 and 7, table 2 only gives the value of $\kappa$ in cases $1,4-6,8$ and 9 . In the shear-driven turbulence (case 9), the value of $\kappa$ is 0.386 , close to that in canonical wall-bounded turbulence (Nagib \& Chauhan 2008; Jiménez 2012; Marusic et al. 2013; Avsarkisov et al. 2014; Lee \& Moser 2015). As shown in table 2, the value of $\kappa$ varies in a narrow range with $0.362 \pm 0.003$, for the Langmuir turbulence considered in the present work, which is slightly smaller than that in the shear-driven turbulence. It was reported in the literature that the value of $\kappa$ is influenced by the boundary geometry (Nagib \& Chauhan 2008; Marusic et al. 2013), body forces such as the buoyancy force (Scagliarini et al. 2015) and the streamwise mean pressure gradient (Luchini 2017). In the Langmuir turbulence, the small discrepancy in the value of $\kappa$ between the shallow-water Langmuir turbulence and shear-driven turbulence is correlated with the $\mathrm{C}-\mathrm{L}$ vortex force, which acts as a body force in the $\mathrm{C}-\mathrm{L}$ equations.

\section{Structure and statistics of velocity fluctuations}

\subsection{Instantaneous velocity fluctuations}

Figure 10 compares the instantaneous velocity fluctuations in an $x-z$ plane at $y / h=0.12$ in the Langmuir turbulence (case 1) with those in the shear-driven turbulence (case 9). The contours of $u^{\prime}$ in the shear-driven turbulence show large-scale streaks with spanwise length scale $5.6 h$, accompanied by smaller-scale streaks (figure 10b1). The contours of $u^{\prime}$ in the Langmuir turbulence are more coherent and mainly dominated by large-scale streaks with spanwise length scale $8 \pi h / 3$ agreeing with those of LCs (figure 10a1), indicating the important contribution of LCs to the streamwise velocity fluctuations. This observation is similar to that of Tejada-Martínez \& Grosch (2007) at the centre of the water column at $R e_{\tau}=395$. Similarly, the comparison between figures $10(a 3)$ and 10(b3) indicates that the 

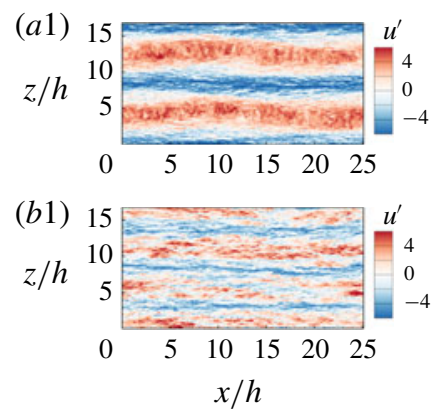

(a2)

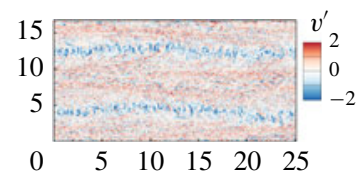

(b2)

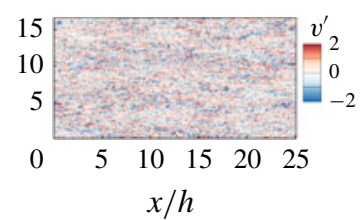

(a3)

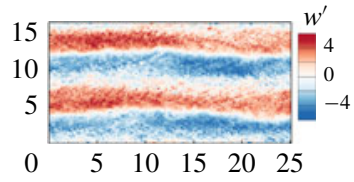

(b3)

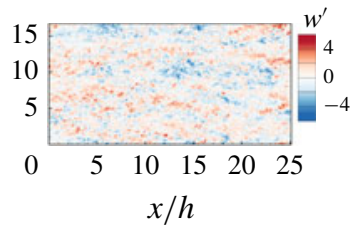

FIGURE 10. (Colour online) Instantaneous velocity fluctuations of (a1) (b1) $u^{\prime}$, (a2) (b2) $v^{\prime}$ and (a3) $(c 3) w^{\prime}$ in a $x-z$ plane at $y / h=0.12$. (a1), (a2) and (a3) are results for case 1 (Langmuir turbulence), while (b1), (b2) and (b3) are for case 9 (shear-driven turbulence).

spanwise velocity fluctuation $w^{\prime}$ is more coherent in the Langmuir turbulence than in the shear-driven turbulence. For the vertical velocity fluctuations, two negative streamwise-elongated streaks with spanwise separation of $8 \pi h / 3$ appear in the Langmuir turbulence (figure 10a2), which are the footprints of the strong downwelling motions of LCs near the bottom. In contrast, the large-scale motion in the shear-driven turbulence is not sufficiently strong to leave footprints in $v^{\prime}$ near the water bottom.

\subsection{Reynolds shear stress}

According to (2.9), the mean velocity $\langle u\rangle$ and Reynolds shear stress $-\left\langle u^{\prime} v^{\prime}\right\rangle$ in the shallow-water Langmuir turbulence are related by the following balance equation of the mean streamwise shear stress

$$
\frac{\mathrm{d}\langle u\rangle^{+}}{\mathrm{d} y^{+}}-\left\langle u^{\prime} v^{\prime}\right\rangle^{+}=1 .
$$

Here, the mean SGS shear stress $\left\langle\tau_{12}^{s g s}\right\rangle$ in (2.9) is neglected, because its magnitude is negligibly small in comparison with $-\left\langle u^{\prime} v^{\prime}\right\rangle$ (figure 3). In this section, the reappearance of the logarithmic layer at $R e_{\tau}=1000$ and the effects of $k h$ and $L a_{t}$ on the thickness of the logarithmic layer are further studied through the analysis of the Reynolds shear stress.

Figure 11(a) compares the profiles of $-\left\langle u^{\prime} v^{\prime}\right\rangle^{+}$in cases 1 and 3 for Langmuir turbulence at $R e_{\tau}=1000$ and 395, respectively, with the result of case 9 for shear-driven turbulence superimposed. Figure 11(b) provides a zoom-in view of their distributions in the logarithmic layer. In all of these three cases, the value of $-\left\langle u^{\prime} v^{\prime}\right\rangle^{+}$ increases monotonically as $y^{+}$increases in the near-bottom region below $y^{+}=100$, and approaches 1.0 in the outer layer above $y^{+}=100$. Substituting equation (4.1) into the definition of $F(3.2)$ gives the relationship between $F$ and $-\left\langle u^{\prime} v^{\prime}\right\rangle^{+}$as

$$
F=y^{+}+y^{+}\left\langle u^{\prime} v^{\prime}\right\rangle^{+} .
$$

According to this equation, although the difference in the profile of $-\left\langle u^{\prime} v^{\prime}\right\rangle^{+}$seems to be small between cases 1 and 3 , it is amplified by the multiplication with $y^{+}$to result in a more significant difference in the profile of $F$.

To further study the effect of the Reynolds shear stress on the logarithmic layer, we depict in figure $11(b)$ the profile of $-\left\langle u^{\prime} v^{\prime}\right\rangle^{+}$in cases 1,3 and 9 in the logarithmic 

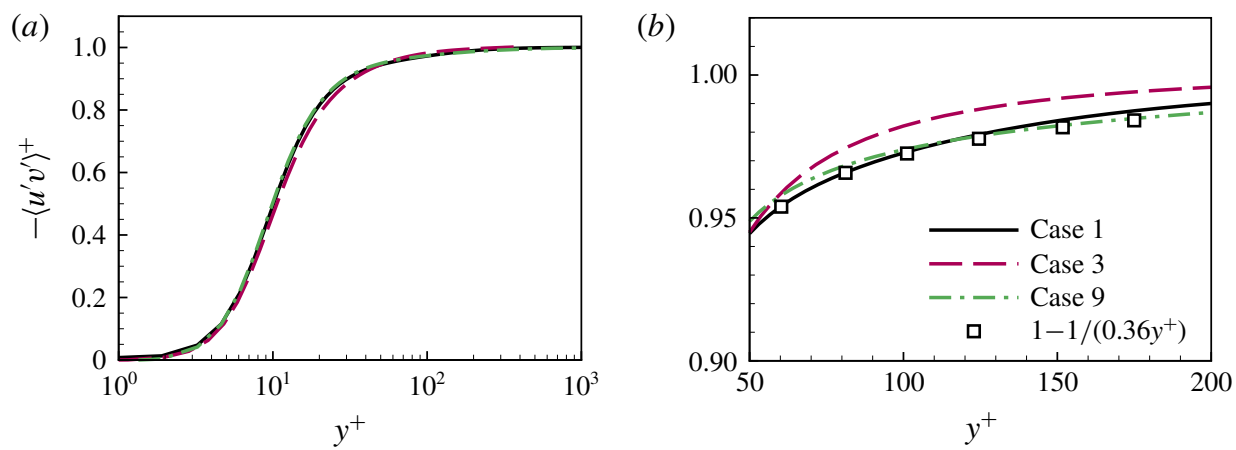

Figure 11. (Colour online) Profiles of $-\left\langle u^{\prime} v^{\prime}\right\rangle^{+}$in cases 1,3 and 9 and comparison with $1-1 /\left(\kappa y^{+}\right)$. LCs are present in cases 1 and 3 with $R e_{\tau}=1000$ and 395, respectively, while LCs are absent in case 9 with $\operatorname{Re}_{\tau}=1000$.
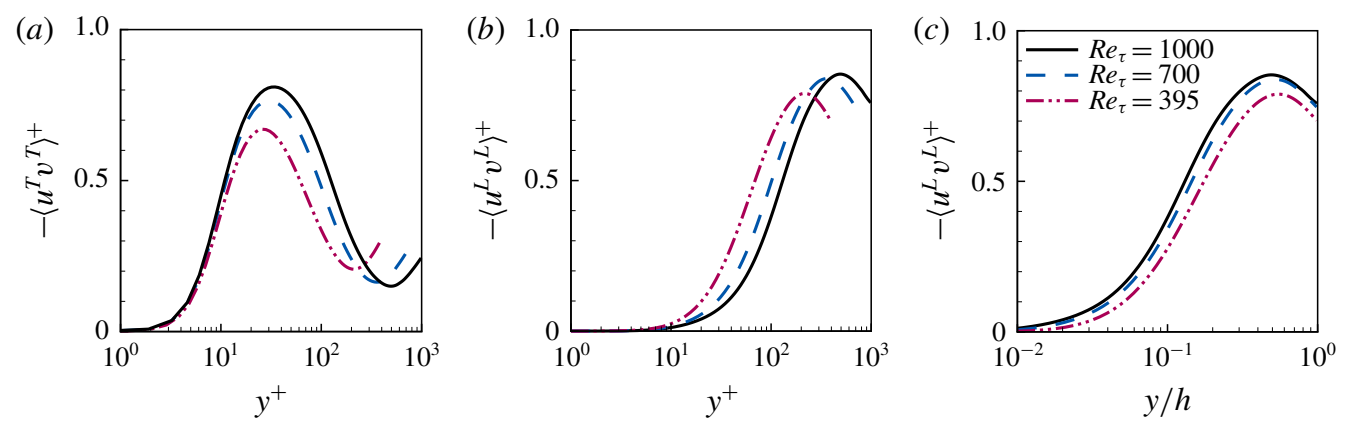

Figure 12. (Colour online) Profiles of $-\left\langle u^{T} v^{T}\right\rangle^{+}$and $-\left\langle u^{L} v^{L}\right\rangle^{+}$in cases 1-3 with $R e_{\tau}=1000,700$ and 395, respectively. (a) Profiles of $-\left\langle u^{T} v^{T}\right\rangle^{+}$in near-bottom coordinate $y^{+}$, and $(b),(c)$ profiles of $-\left\langle u^{L} v^{L}\right\rangle^{+}$in near-bottom coordinate $y^{+}$and water-column coordinate $y / h$, respectively.

layer of the shear-driven turbulence between $y^{+}=50$ and 200. According to (4.1), the identity $-\left\langle u^{\prime} v^{\prime}\right\rangle^{+}=1-1 /\left(k y^{+}\right)$holds in the logarithmic layer. Figure 11(b) shows that in case 3 for Langmuir turbulence at $R e_{\tau}=395$, the value of $-\left\langle u^{\prime} v^{\prime}\right\rangle^{+}$is larger than $1-1 /\left(k y^{+}\right)$, indicating that the mean velocity deviates from the logarithmic law. In contrast, in case 1 for Langmuir turbulence at $R e_{\tau}=1000$, the profile of $-\left\langle u^{\prime} v^{\prime}\right\rangle^{+}$is close to that of $1-1 /\left(k y^{+}\right)$, meaning that the logarithmic layer reappears.

The effect of LCs on the Reynolds shear stress can be further assessed through the decomposition of $-\left\langle u^{\prime} v^{\prime}\right\rangle^{+}$into the LC-coherent part $-\left\langle u^{L} v^{L}\right\rangle^{+}$and the residual part $-\left\langle u^{T} v^{T}\right\rangle^{+}$(2.11). Figures $12(a)$ and 12(b) compare respectively the profiles of $-\left\langle u^{T} v^{T}\right\rangle^{+}$and $-\left\langle u^{L} v^{L}\right\rangle^{+}$in cases 1-3 in the near-bottom coordinate $y^{+}$, while figure $12(c)$ compares the profile of $-\left\langle u^{L} v^{L}\right\rangle^{+}$in these cases in the water-column coordinate $y / h$ to further study the effect of the Reynolds number on the Reynolds shear stress. From the comparison between figures $12(a)$ and $12(b)$, it can be observed that the residual part $-\left\langle u^{T} v^{T}\right\rangle^{+}$is dominant below $y^{+}=100$, while the LC-coherent part $-\left\langle u^{L} v^{L}\right\rangle^{+}$is more important above $y^{+}=100$, consistent with the observations of Tejada-Martínez \& Grosch (2007) and Sinha et al. (2015). Figure 12(a) shows that, as the Reynolds number increases, the peak location of $-\left\langle u^{T} v^{T}\right\rangle^{+}$remains almost unchanged in the near-bottom coordinate. Different from $-\left\langle u^{T} v^{T}\right\rangle^{+}$, the peaks of 

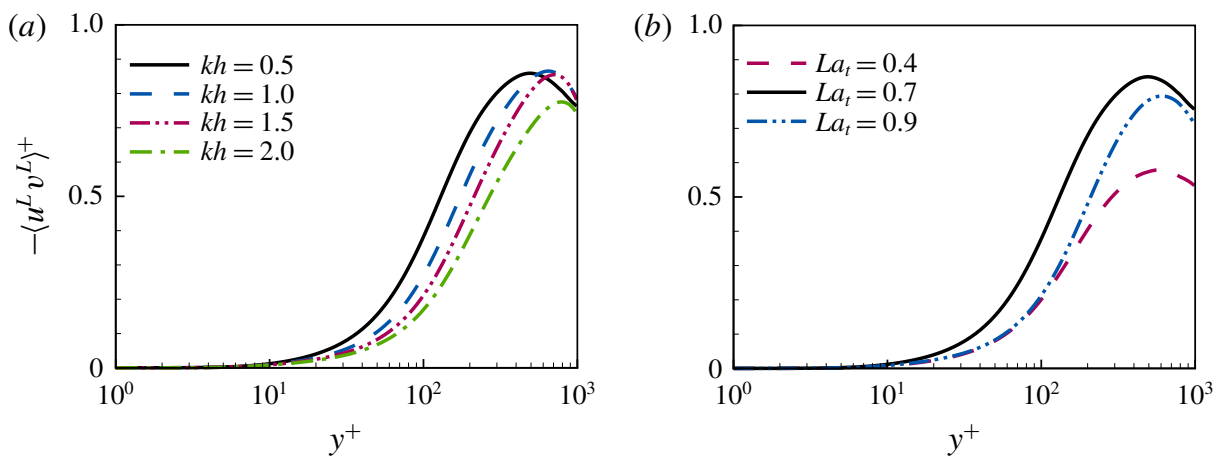

Figure 13. (Colour online) Profiles of $-\left\langle u^{L} v^{L}\right\rangle^{+}$in $(a)$ cases 1 and 4-6 with $k h=0.5,1$, 1.5 and 2, respectively, and $(b)$ cases 1,7 and 8 with $L a_{t}=0.7,0.4$ and 0.9 , respectively.

$-\left\langle u^{L} v^{L}\right\rangle^{+}$are collocated in the water-column coordinate at $y / h=0.5$ (figure $12 c$ ). Note that the near-bottom and water-column coordinates are related by the Reynolds number as $y^{+}=R e_{\tau} y / h$. As a result, the peaks of $-\left\langle u^{L} v^{L}\right\rangle^{+}$collocated at the same value of $y / h$ correspond to an increasing value of $y^{+}$as the Reynolds number increases (figure 12b). This indicates a weaker influence of LCs on the momentum transfer near the water bottom at higher Reynolds number, characterized by the smaller magnitude of $-\left\langle u^{L} v^{L}\right\rangle^{+}$below $y^{+}=200$ (figure 12b). This effect of Reynolds number leads to the reappearance of the logarithmic layer in the near-bottom region at $R e_{\tau}=1000$ (figure 7).

Figure 13 shows the effects of $k h$ and $L a_{t}$ on the LC-coherent Reynolds shear stress $-\left\langle u^{L} v^{L}\right\rangle^{+}$. As depicted in figure 13(a), as the value of $k h$ increases, the peak of $-\left\langle u^{L} v^{L}\right\rangle^{+}$shifts to larger $y^{+}$, and the magnitude of $-\left\langle u^{L} v^{L}\right\rangle^{+}$decreases below $y^{+}=$ 200. This indicates a weaker effect of LCs on the momentum transfer in the nearbottom region at larger value of $k h$. As a result, the logarithmic layer expands as the value of $k h$ increases (figure 8).

The magnitude of $-\left\langle u^{L} v^{L}\right\rangle^{+}$is non-monotonic with the increase of $L a_{t}$. As shown in figure $13(b)$, the magnitude of $-\left\langle u^{L} v^{L}\right\rangle^{+}$at $L a_{t}=0.7$ is larger than those at $L a_{t}=0.4$ and 0.9 between $y^{+}=20$ and 1000. The larger magnitude of $-\left\langle u^{L} v^{L}\right\rangle^{+}$at $L a_{t}=0.7$ than at $L a_{t}=0.9$ can be attributed to the stronger intensity of LCs due to the larger magnitude of vortex force at smaller value of $L a_{t}(2.2)$. The magnitude of $-\left\langle u^{L} v^{L}\right\rangle^{+}$is smaller at $L a_{t}=0.4$ than at $L a_{t}=0.7$, indicating a less important contribution of LCs to the momentum transfer at $L a_{t}=0.4$, which is consistent with the observation of Sinha et al. (2015) at $R e_{\tau}=395$. As explained by Sinha et al. (2015), the intensities of the upwelling motion induced by LCs become stronger at a smaller value of $L a_{t}$ due to the larger magnitude of $\mathrm{C}-\mathrm{L}$ vortex forcing. The strong upwelling motion at $L a_{t}=0.4$ shifts LCs towards the water surface. As a result, the magnitude of the LC-coherent Reynolds stress is smaller at $L a_{t}=0.4$ than at $L a_{t}=0.7$ in the lower half of the water column. The residual turbulence motions become important in the momentum transfer at $L a_{t}=0.4$, which is indicated by $-\left\langle u^{T} v^{T}\right\rangle^{+}$. To further study the effects of $L a_{t}$ on the residual turbulence motions, we compare $-\left\langle u^{T} v^{T}\right\rangle^{+}$among cases 1,7 and 8 in figure 14(a), and the pre-multiplied spanwise co-spectrum $\phi_{u v}^{T}\left(y, k_{z}\right)=k_{z} E_{u v}^{T}\left(y, k_{z}\right)$ at a near-bottom plane $y^{+}=120$ and a water-column central plane $y / h=1.0$ in figures $14(b)$ and $14(c)$, respectively. Here, $k_{z}=2 \pi / \lambda_{z}$ is the spanwise wavenumber corresponding to the spanwise length scale 

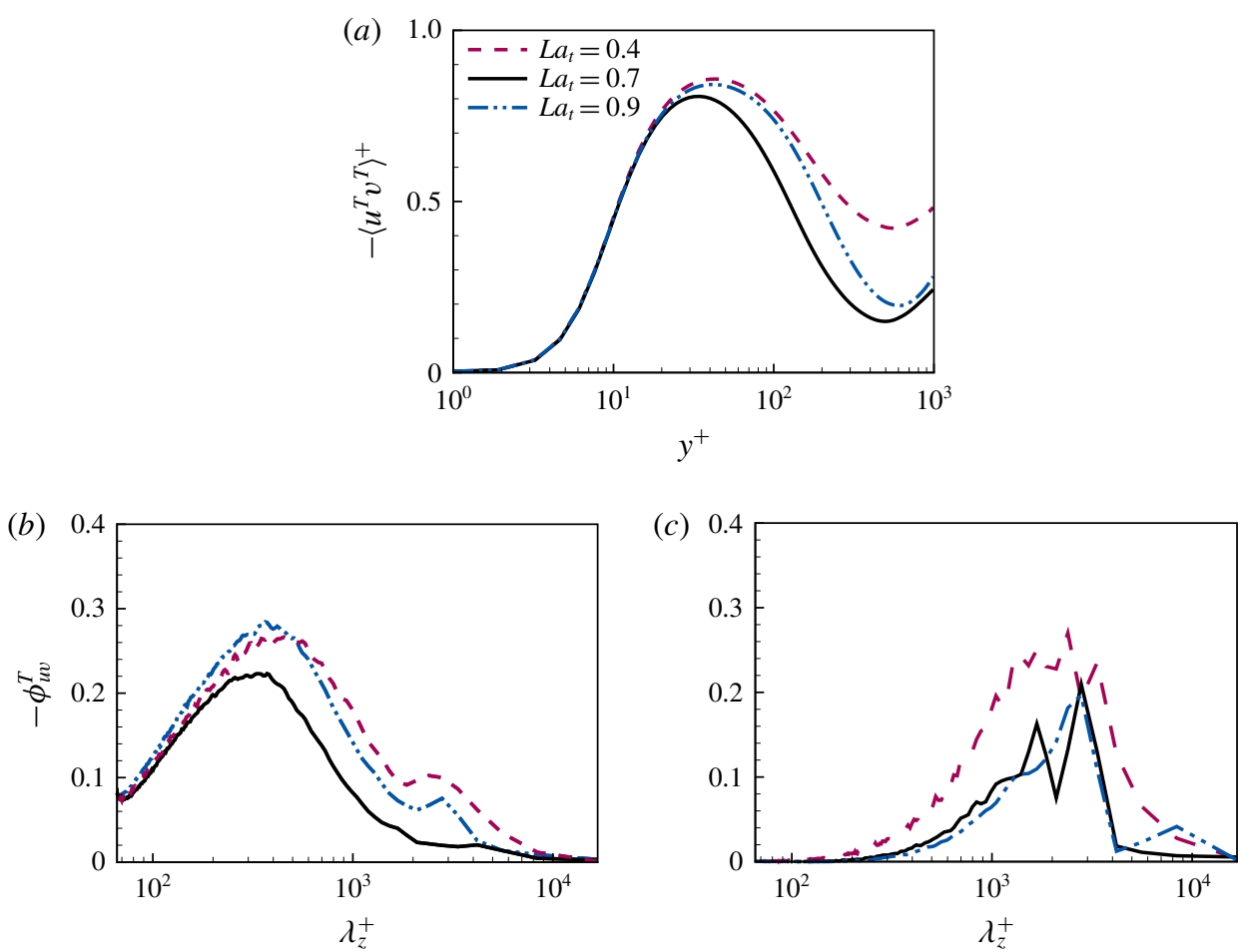

FIgURE 14. (Colour online) Residual part of Reynolds shear stress $-\left\langle u^{T} v^{T}\right\rangle^{+}$and pre-multiplied co-spectrum $-\phi_{u v}^{T}$ in cases 1,7 and 8 with $L a_{t}=0.7,0.4$ and 0.9 , respectively. (a) Profiles of $-\left\langle u^{T} v^{T}\right\rangle^{+},(b)$ pre-multiplied co-spectra $-\phi_{u v}^{T}$ at $y^{+}=120$ and (c) pre-multiplied co-spectra $-\phi_{u v}^{T}$ at $y / h=1$.

$\lambda_{z}$, and $E_{u v}^{T}\left(y, k_{z}\right)$ is the spanwise co-spectrum between $u^{T}$ and $v^{T}$, which satisfies the normalization property

$$
\left\langle u^{T} v^{T}\right\rangle(y)=\int_{0}^{\infty} E_{u v}^{T}\left(y, k_{z}\right) \mathrm{d} k_{z}=\int_{-\infty}^{+\infty} k_{z} E_{u v}^{T}\left(y, k_{z}\right) \mathrm{d} \log \left(k_{z}\right) .
$$

As shown in figure 14(a), the magnitude of the residual turbulence part of Reynolds shear stress $-\left\langle u^{T} v^{T}\right\rangle^{+}$is larger at $L a_{t}=0.4$ than at $L a_{t}=0.7$ above $y^{+}=20$ and $L a_{t}=0.9$ above $y^{+}=100$, which results from the intensification of residual turbulence motions. The effect of turbulence motions on $-\left\langle u^{T} v^{T}\right\rangle^{+}$can be observed from the pre-multiplied spanwise co-spectrum $-\phi_{u v}^{T}$ shown in figure $14(b, c)$. At the near-bottom plane $y^{+}=120$ (figure $14 b$ ), the magnitude of $-\phi_{u v}^{T}$ at $L a_{t}=0.4$ is larger than that at $L a_{t}=0.7$ for $\lambda_{z}^{+}>200$, indicating that the larger magnitude of $-\left\langle u^{T} v^{T}\right\rangle^{+}$at $L a_{t}=0.4$ results from the enhancement of the large-scale turbulence motions $\left(\lambda_{z}^{+}>200\right)$. Akan et al. (2013) and Sinha et al. (2015) pointed out that the strong shear of Stokes drift at $L a_{t}=0.4$ significantly enhances the large-scale turbulent motion in the vertical direction in the upper half of the water column. It is evident from figure $14(c)$ that the large-scale turbulent motions also lead to the magnitude increase of $-\phi_{u v}^{T}$ at spanwise scales larger than $\lambda_{z}^{+}=200$ at the centre of the water column $y / h=1$. By comparing figure $14(b)$ with figure $14(c)$, it is observed that as the value of $L a_{t}$ decreases from 0.7 to 0.4 , the magnitude of $-\phi_{u v}^{T}$ increases at the same spanwise scales $\left(\lambda_{z}^{+}>200\right)$. 

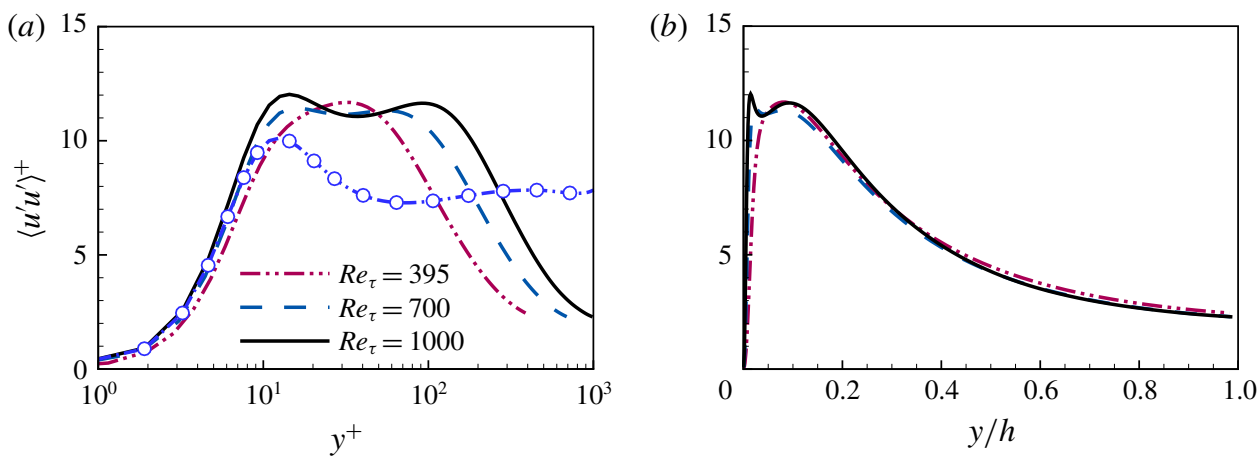

FIgURE 15. (Colour online) Profiles of $\left\langle u^{\prime} u^{\prime}\right\rangle^{+}$in the coordinate of $(a) y^{+}$and $(b) y / h$ in cases 1-3 with $R e_{\tau}=1000,700$ and 395, respectively. In $(a)$, the line with open circles represents the result in case 9 (the shear-driven turbulence at $R e_{\tau}=1000$ ) as a reference.

This indicates that the large-scale turbulent motions are sufficiently strong to reach the near-bottom region. As a result, the magnitude of $-\left\langle u^{T} v^{T}\right\rangle$ also becomes stronger at $L a_{t}=0.4$ than at $L a_{t}=0.7$ near the water bottom. This effect of large-scale turbulence motion on the near-bottom momentum flux is absent at larger value of $L a_{t}$, because of the smaller Stokes shear. As shown in figure $14(b)$, the magnitude of $-\phi_{u v}^{T}$ is larger at $L a_{t}=0.9$ than at $L a_{t}=0.7$ at all spanwise scales $\lambda_{z}^{+}$. In contrast, as shown in figure $14(c)$, at $y / h=1$, the magnitudes of $-\phi_{u v}^{T}$ at $L a_{t}=0.9$ and 0.7 are comparable at most spanwise scales except for $\lambda_{z}^{+} \geqslant 7000$. This indicates that the suppression of $-\left\langle u^{T} v^{T}\right\rangle^{+}$near the water bottom in response to the decrease of $L a_{t}$ from 0.9 to 0.7 cannot be attributed to large-scale turbulence motions at $y / h=1$. Martinat et al. (2014) and Sinha et al. (2015) pointed out that LCs tend to suppress the momentum flux induced by turbulent motions. Because the impact of LCs on the momentum flux near the bottom is weaker at $L a_{t}=0.9$ than at $L a_{t}=0.7$ (figure $13 b$ ), the magnitude of $-\left\langle u^{T} v^{T}\right\rangle^{+}$becomes larger as $L a_{t}$ increases from 0.7 to 0.9 .

\subsection{Reynolds normal stresses}

We start our analyses of Reynolds normal stresses with the effect of the Reynolds number. Figure 15 compares the profiles of $\left\langle u^{\prime} u^{\prime}\right\rangle^{+}$among cases 1-3 with different Reynolds numbers. The result of case 9 for shear-driven turbulence is superimposed for comparison. As shown in figure $15(a)$, at $R e_{\tau}=395$, there is a single peak in the profile of $\left\langle u^{\prime} u^{\prime}\right\rangle^{+}$. This peak is located at $y^{+}=32$, different from the peak location of $\left\langle u^{\prime} u^{\prime}\right\rangle^{+}$at $y^{+}=11$ in the shear-driven turbulence. As the Reynolds number increases to 700 and 1000, the profile of $\left\langle u^{\prime} u^{\prime}\right\rangle^{+}$shows a bimodal shape. As shown in figure 15(a), the inner peak at $y^{+}=12$ is close to that in the shear-driven turbulence. Although an outer peak of $\left\langle u^{\prime} u^{\prime}\right\rangle^{+}$also occurs in canonical wall-bounded turbulence for $R e_{\tau}>$ 15000 (Alfredsson, Segalini \& Örlü 2011), the one in Langmuir turbulence is different in three aspects. First, the outer peak of $\left\langle u^{\prime} u^{\prime}\right\rangle^{+}$in the Langmuir turbulence occurs at a much smaller Reynolds number of 700. Second, in the shallow-water Langmuir turbulence, the outer peak location is scaled by $h$ for various Reynolds numbers. As shown in figure $15(b)$, at $R e_{\tau}=700$ and 1000 , the outer peak of $\left\langle u^{\prime} u^{\prime}\right\rangle^{+}$is collocated at $y / h=0.11$. However, the outer peak in the canonical wall-bounded turbulence for $R e_{\tau}>15000$ is located at $y^{+} \sim \operatorname{Re}_{\tau}^{1 / 2}$ (Alfredsson et al. 2011). Third, as shown in figure $15(b)$, in the water-column coordinate $y / h$, the profiles of $\left\langle u^{\prime} u^{\prime}\right\rangle^{+}$in the outer 

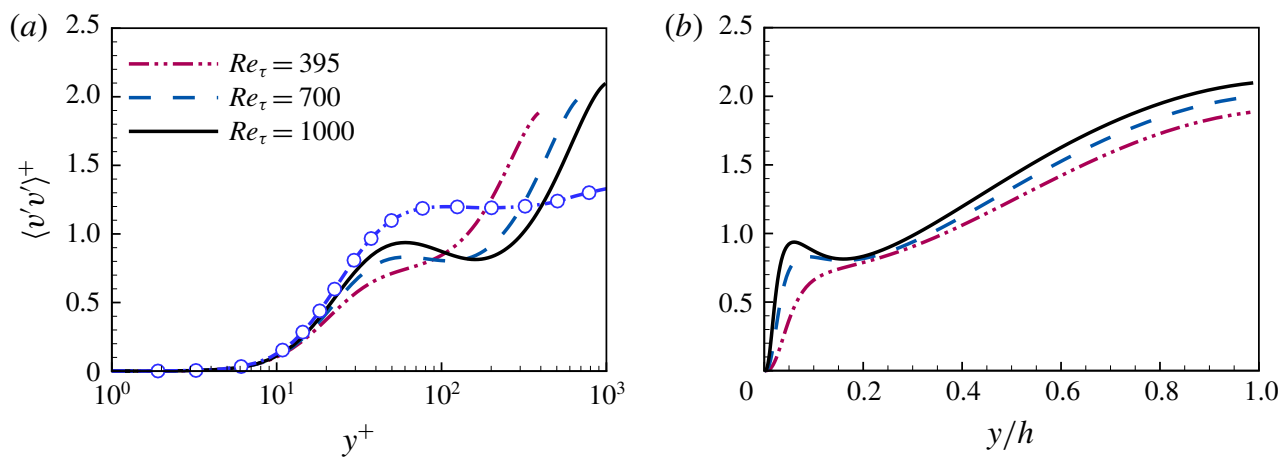

Figure 16. (Colour online) Profiles of $\left\langle v^{\prime} v^{\prime}\right\rangle^{+}$in the coordinate of $(a) y^{+}$and $(b) y / h$ in cases 1-3 with $R e_{\tau}=1000,700$ and 395, respectively. In $(a)$, the line with open circles represents the result in case 9 (the shear-driven turbulence at $R e_{\tau}=1000$ ) as a reference.

layer of the Langmuir turbulence at various Reynolds numbers are close to each other. In contrast, in the canonical wall-bounded turbulence, the profiles of $\left\langle u^{\prime} u^{\prime}\right\rangle^{+}$at various Reynolds numbers deviate from each other in the outer coordinate $y / h$ (Hoyas \& Jiménez 2006; Avsarkisov et al. 2014; Bernardini et al. 2014; Lee \& Moser 2015). It is evident from the above three points that the occurrence of LCs significantly alters the profile of $\left\langle u^{\prime} u^{\prime}\right\rangle^{+}$.

Figure 16 shows the Reynolds number effect on the profiles of $\left\langle v^{\prime} v^{\prime}\right\rangle^{+}$. As shown in figure 16(a), the value of $\left\langle v^{\prime} v^{\prime}\right\rangle^{+}$increases monotonically with $y^{+}$at $R e_{\tau}=395$. In contrast, at $R e_{\tau}=700$ and 1000 , a local peak located at $y^{+}=50$ occurs in the profile of $\left\langle v^{\prime} v^{\prime}\right\rangle^{+}$. As the distance from the bottom further increases, the profiles of $\left\langle v^{\prime} v^{\prime}\right\rangle^{+}$at $R e_{\tau}=700$ and 1000 first reach their local minimal values at $y / h \approx 0.17$, and then increases again with $y / h$ (figure 16b). The shape of the $\left\langle v^{\prime} v^{\prime}\right\rangle^{+}$profile in shallow-water Langmuir turbulence is drastically different from that in the shear-driven turbulence and canonical wall-bounded turbulence (Kim, Moin \& Moser 1987; Hoyas \& Jiménez 2006; Avsarkisov et al. 2014; Bernardini et al. 2014; Lee \& Moser 2015). For example, the value of $\left\langle v^{\prime} v^{\prime}\right\rangle^{+}$in the turbulent Couette flow (Avsarkisov et al. 2014) and the shear-driven turbulence is close to a constant in the outer layer, while in the pressure-driven turbulent channel flow the magnitude of $\left\langle v^{\prime} v^{\prime}\right\rangle^{+}$decreases with $y^{+}$in the outer layer (Kim et al. 1987; Hoyas \& Jiménez 2006; Lee \& Moser 2015).

Figure 17 compares the profiles of $\left\langle w^{\prime} w^{\prime}\right\rangle^{+}$in shallow-water Langmuir turbulence at various Reynolds numbers. Figure $17(a)$ shows that in the wall coordinate $y^{+}$, the magnitude of $\left\langle w^{\prime} w^{\prime}\right\rangle^{+}$increases as the Reynolds number increases. In Langmuir turbulence at $R e_{\tau}=395,700$ and 1000, the peak value of $\left\langle w^{\prime} w^{\prime}\right\rangle^{+}$is respectively 5.7, 6.6 and 7.2, significantly larger than those in the shear-driven turbulence (figure 17a) and canonical wall-bounded turbulence at $R e_{\tau} \leqslant 1000$ (approximately 2.0-3.0, see e.g. Kim et al. 1987; Hoyas \& Jiménez 2006; Avsarkisov et al. 2014; Bernardini et al. 2014). The peak location of $\left\langle w^{\prime} w^{\prime}\right\rangle^{+}$in Langmuir turbulence is also different from that in shear-driven turbulence and canonical wall-bounded turbulence. In Langmuir turbulence, the peak location of $\left\langle w^{\prime} w^{\prime}\right\rangle^{+}$is scaled by $h$. As shown in figure $17(b)$, the peaks of $\left\langle w^{\prime} w^{\prime}\right\rangle^{+}$at various Reynolds numbers collocate at $y / h=0.15$. In canonical wall-bounded turbulence, the peak of $\left\langle w^{\prime} w^{\prime}\right\rangle^{+}$is located at $y^{+}=30-40$ (Kim et al. 1987; Hoyas \& Jiménez 2006; Avsarkisov et al. 2014; Bernardini et al. 2014), closer to the wall than in Langmuir turbulence. Similarly, in the shear-driven turbulence the 

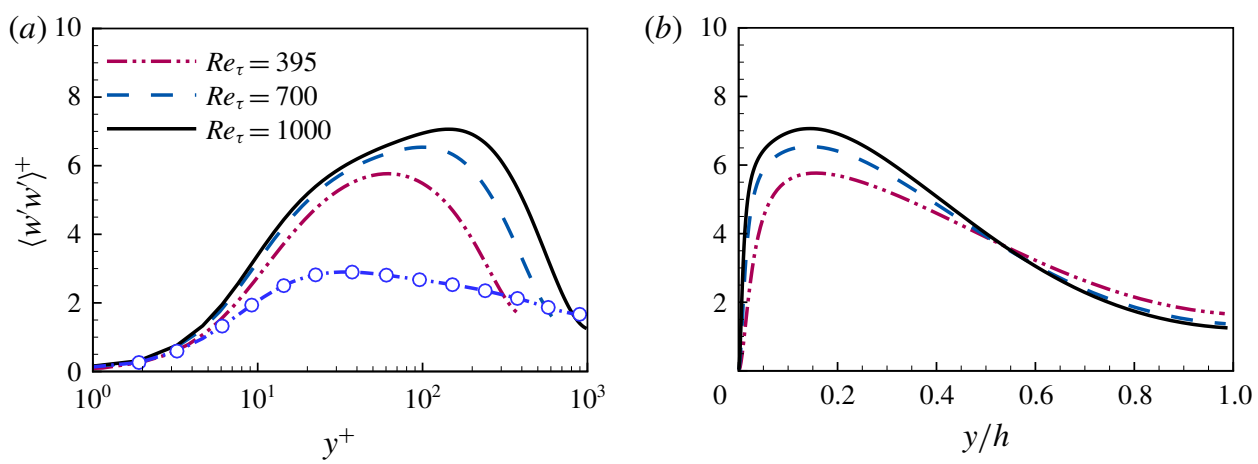

FIgURE 17. (Colour online) Profiles of $\left\langle w^{\prime} w^{\prime}\right\rangle^{+}$in the coordinate of $(a) y^{+}$and $(b) y / h$ in cases 1-3 with $R e_{\tau}=1000,700$ and 395, respectively. In $(a)$, the line with open circles represents the result in case 9 (the shear-driven turbulence at $R e_{\tau}=1000$ ) as a reference.
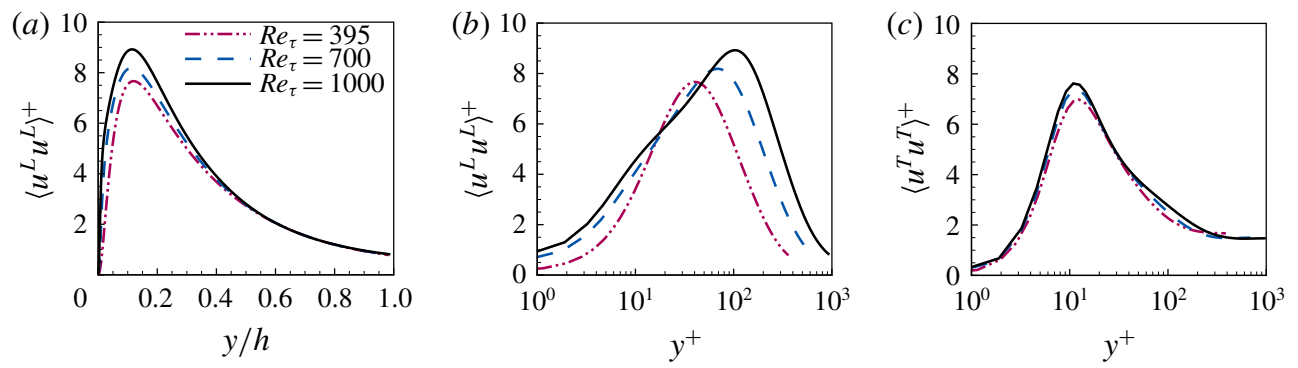

FIgURE 18. (Colour online) Profiles of $(a)\left\langle u^{L} u^{L}\right\rangle^{+}$in the coordinate of $y / h,(b)\left\langle u^{L} u^{L}\right\rangle^{+}$ and $(c)\left\langle u^{T} u^{T}\right\rangle^{+}$in the coordinate of $y^{+}$in cases 1-3 with $R e_{\tau}=1000,700$ and 395, respectively.

peak is located at $y^{+}=30$ (figure 17a). From the comparison of the peak magnitude and location of $\left\langle u^{\prime} u^{\prime}\right\rangle^{+},\left\langle v^{\prime} v^{\prime}\right\rangle^{+}$and $\left\langle w^{\prime} w^{\prime}\right\rangle^{+}$between the shallow-water Langmuir turbulence and canonical wall turbulence without LCs, it is evident that LCs make a substantial contribution to the Reynolds normal stresses in Langmuir turbulence.

Similar to the analyses of Reynolds shear stress in $\$ 4.2$, we employ the decomposition of the Reynolds stress (2.11) to study the effect of LCs on the Reynolds normal stresses in Langmuir turbulence at various Reynolds numbers. Figure 18 shows the profiles of $\left\langle u^{L} u^{L}\right\rangle^{+}$and $\left\langle u^{T} u^{T}\right\rangle^{+}$, while figures 19 and 20 show respectively those of $\left\langle v^{L} v^{L}\right\rangle^{+}$and $\left\langle v^{T} v^{T}\right\rangle^{+}$and those of $\left\langle w^{L} w^{L}\right\rangle^{+}$and $\left\langle w^{T} w^{T}\right\rangle^{+}$at various Reynolds numbers. In each of these three figures, the left column compares the profiles of LC-coherent part in the water-column coordinate, while the middle and right columns compare respectively the profiles of LC-coherent and residual parts in the near-bottom coordinate.

From the comparison among figures 15-20, it can be observed that in the outer layer, all of the three LC-coherent Reynolds normal stresses make important contributions to the total Reynolds normal stresses, which is consistent with the observations of Tejada-Martínez \& Grosch (2007) and Sinha et al. (2015) at $R e_{\tau}=395$. Figure 18(a) shows that the location of the $\left\langle u^{L} u^{L}\right\rangle^{+}$peak is scaled by $h$. The peaks of $\left\langle u^{L} u^{L}\right\rangle^{+}$at various Reynolds numbers collocate at $y / h=0.11$, which is consistent with the outer peak of $\left\langle u^{\prime} u^{\prime}\right\rangle^{+}$at $R e_{\tau}=700$ and 1000 (figure 15b). From the comparison 

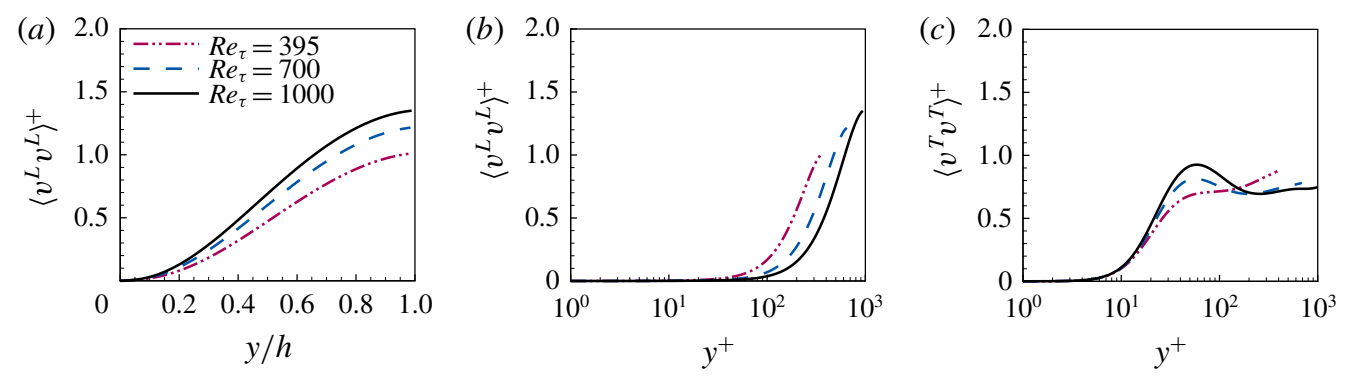

FIgURE 19. (Colour online) Profiles of $(a)\left\langle v^{L} v^{L}\right\rangle^{+}$in the coordinate of $y / h,(b)\left\langle v^{L} v^{L}\right\rangle^{+}$ and $(c)\left\langle v^{T} v^{T}\right\rangle^{+}$in the coordinate of $y^{+}$in cases 1-3 with $R e_{\tau}=1000,700$ and 395, respectively.
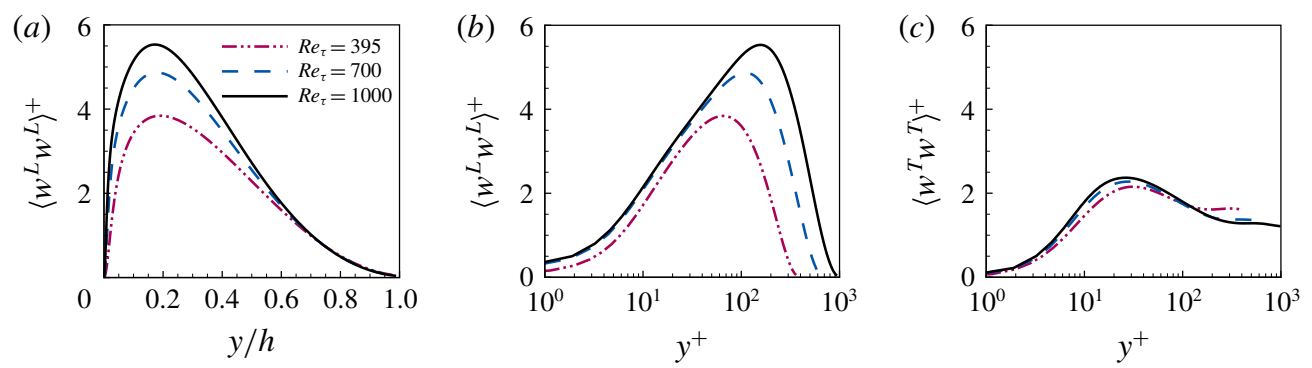

Figure 20. (Colour online) Profiles of $(a)\left\langle w^{L} w^{L}\right\rangle^{+}$in the coordinate of $y / h,(b)\left\langle w^{L} w^{L}\right\rangle^{+}$ and $(c)\left\langle w^{T} w^{T}\right\rangle^{+}$in the coordinate of $y^{+}$in cases 1-3 with $R e_{\tau}=1000,700$ and 395, respectively.

between figures $18(b)$ and $18(c)$, it can be observed that the magnitude of $\left\langle u^{L} u^{L}\right\rangle^{+}$ is much larger than that of $\left\langle u^{T} u^{T}\right\rangle^{+}$above $y^{+}=100$, indicating that the outer peaks of $\left\langle u^{\prime} u^{\prime}\right\rangle^{+}$at $R e_{\tau}=700$ and 1000 are induced by the LCs. Figure 19(b) shows that the value of $\left\langle v^{L} v^{L}\right\rangle^{+}$increases with $y^{+}$in the outer layer. In contrast, as depicted in figure $19(c)$, the profile of $\left\langle v^{T} v^{T}\right\rangle^{+}$is relatively flat above $y^{+}=100$. This indicates that the increase of $\left\langle v^{\prime} v^{\prime}\right\rangle^{+}$with $y^{+}$above $y^{+}=100$ (figure 16b) is caused by the LCs. Similar to $\left\langle u^{L} u^{L}\right\rangle^{+}$, the peak location of $\left\langle w^{L} w^{L}\right\rangle^{+}$is scaled by $h$ at various Reynolds numbers. As shown in figure $20(a)$, the peaks of $\left\langle w^{L} w^{L}\right\rangle^{+}$at various Reynolds numbers collocate at $y / h=0.16$, which are close to those of $\left\langle w^{\prime} w^{\prime}\right\rangle^{+}$at $y / h=0.15$ (figure 17b). In contrast, as shown in figure $20(c)$, the magnitude of the $\left\langle w^{T} w^{T}\right\rangle^{+}$ profile changes little with $y^{+}$. Therefore, the peak of $\left\langle w^{\prime} w^{\prime}\right\rangle^{+}$in the shallow-water Langmuir turbulence results from the contribution of LCs.

In the near-bottom region below $y^{+}=100$, it can be observed from the comparisons between figures $18(b)$ and $18(c)$, and between figures $20(b)$ and $20(c)$ that the magnitudes of $\left\langle u^{L} u^{L}\right\rangle^{+}$and $\left\langle w^{L} w^{L}\right\rangle^{+}$are comparable to those of $\left\langle u^{T} u^{T}\right\rangle^{+}$and $\left\langle w^{T} w^{T}\right\rangle^{+}$, respectively. This indicates that both the LC-coherent and residual parts are important to the total Reynolds normal stresses $\left\langle u^{\prime} u^{\prime}\right\rangle$ and $\left\langle w^{\prime} w^{\prime}\right\rangle$ near the bottom. As shown in figure $18(c)$, the peaks of $\left\langle u^{T} u^{T}\right\rangle^{+}$at various Reynolds numbers collocate at $y^{+}=12$. At $R e_{\tau}=395$, because the peak of $\left\langle u^{T} u^{T}\right\rangle^{+}$is close to that of $\left\langle u^{L} u^{L}\right\rangle^{+}$, neither of them alone is shown in the profile of the total Reynolds normal stress $\left\langle u^{\prime} u^{\prime}\right\rangle$ (figure $15 a$ ). As the Reynolds number increases, the peak of $\left\langle u^{T} u^{T}\right\rangle^{+}$is located at the same $y^{+}$, but that of $\left\langle u^{L} u^{L}\right\rangle^{+}$moves to larger $y^{+}$. As a result, the peaks of both $\left\langle u^{T} u^{T}\right\rangle^{+}$and $\left\langle u^{L} u^{L}\right\rangle$ 

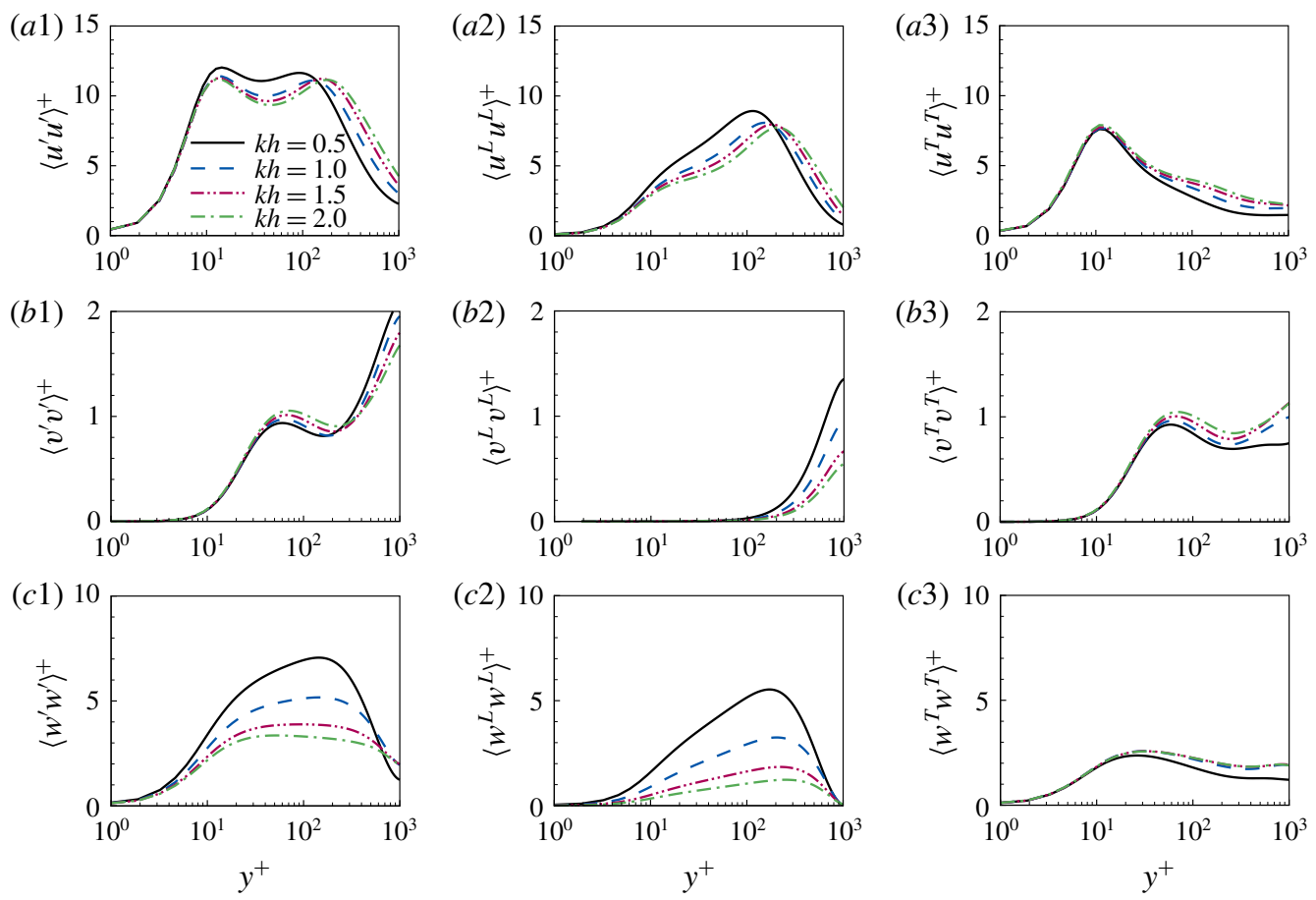

FIGURE 21. (Colour online) Profiles of $(a 1)\left\langle u^{\prime} u^{\prime}\right\rangle^{+}$, (a2) $\left\langle u^{L} u^{L}\right\rangle^{+}$, (a3) $\left\langle u^{T} u^{T}\right\rangle^{+}$, (b1) $\left\langle v^{\prime} v^{\prime}\right\rangle^{+}$, (b2) $\left\langle v^{L} v^{L}\right\rangle^{+}$, (b3) $\left\langle v^{T} v^{T}\right\rangle^{+}$, (c1) $\left\langle w^{\prime} w^{\prime}\right\rangle^{+}$, (c2) $\left\langle w^{L} w^{L}\right\rangle^{+}$and $(c 3)\left\langle w^{T} w^{T}\right\rangle^{+}$in cases 1 and $4-6$ with $k h=0.5,1,1.5$ and 2 , respectively.

are shown in the profile of $\left\langle u^{\prime} u^{\prime}\right\rangle$, forming a bimodal shape (figure 15a). The peak of $\left\langle w^{T} w^{T}\right\rangle^{+}$for various Reynolds numbers collocates at $y^{+}=30$, where its magnitude is smaller than that of $\left\langle w^{L} w^{L}\right\rangle^{+}$. As a result, the peak of $\left\langle w^{T} w^{T}\right\rangle^{+}$is not shown in the profile of $\left\langle w^{\prime} w^{\prime}\right\rangle$ (figure 17a). From the comparison between figure $17(a)$ and figure $20(b)$, it can be deduced that the single peak of $\left\langle w^{\prime} w^{\prime}\right\rangle$ is induced by the LC-coherent part $\left\langle w^{L} w^{L}\right\rangle^{+}$. The peak locations of $\left\langle w^{\prime} w^{\prime}\right\rangle$ and $\left\langle w^{L} w^{L}\right\rangle^{+}$are close to each other. As the Reynolds number increases, they both shift away from the bottom, with their magnitude increasing.

Different from $\left\langle u^{L} u^{L}\right\rangle^{+}$and $\left\langle w^{L} w^{L}\right\rangle^{+}$, the magnitude of $\left\langle v^{L} v^{L}\right\rangle^{+}$is much smaller than that of $\left\langle v^{T} v^{T}\right\rangle^{+}$near the water bottom (figure 19b,c). In wall-bounded turbulent flows, the vertical mixing is responsible for the generation of Reynolds shear stresses (Blackwelder \& Eckelmann 1979; Robinson 1991; Jeong et al. 1997; Jiménez \& Pinelli 1999; Panton 2001). As a result, the magnitude of $-\left\langle u^{L} v^{L}\right\rangle^{+}$is also small in the near-bottom region (figure 12b). As the Reynolds number increases, the profile of $\left\langle v^{L} v^{L}\right\rangle^{+}$shifts to larger $y^{+}$, leading to the thickness increase of the near-bottom region with a small magnitude of $\left\langle v^{L} v^{L}\right\rangle^{+}$. In response, the near-bottom region with a small magnitude of $-\left\langle u^{L} v^{L}\right\rangle^{+}$also becomes thicker at higher Reynolds number, which in turn leads to the reappearance of the logarithmic layer at $R e_{\tau}=1000$.

Figure 21 shows the effects of $k h$ on the Reynolds normal stresses, together with their LC-coherent parts and the residual turbulence parts. As the value of $k h$ increases, the profile of Stokes drift velocity in (2.4) shifts towards the water surface, and the magnitude of vortex force term in the $\mathrm{C}-\mathrm{L}$ equations (2.2) is suppressed in the bottom half of the water column, which weakens the intensity of LCs. In 
response, the profiles of the LC-coherent Reynolds normal stresses shift towards the water surface (figure $21 a 2, b 2, c 2$ ), with the magnitude of the $\left\langle u^{L} u^{L}\right\rangle^{+}$peak decreasing slightly (figure 21a2) and those of $\left\langle v^{L} v^{L}\right\rangle^{+}$and $\left\langle w^{L} w^{L}\right\rangle^{+}$decreasing throughout the bottom half of the water column (figure 21b2,c2). The present observation of the effects of $k h$ on the intensity of LCs is consistent with that of Tejada-Martínez et al. (2012) and Sinha et al. (2015) at $\operatorname{Re}_{\tau}=395$. The smaller magnitude of $\left\langle v^{L} v^{L}\right\rangle^{+}$ indicates weaker vertical turbulence mixing coherent to the motion of LCs, such that the magnitude of $-\left\langle u^{L} v^{L}\right\rangle^{+}$decreases as the value of $k h$ increases (figure 13a), resulting in the wider logarithmic layer at larger value of $k h$ (figure 8).

In the outer layer, because the turbulence is dominated by the LCs, the effects of $k h$ on the Reynolds normal stresses are dominated by those on their LC-coherent parts. This is evident by comparing figures 21(a1), 21(b1), 21(c1) with figures 21(a2), 21(b2) and 21(c2), respectively. From the comparison, it can be observed that above $y^{+}=200$, the effects of $k h$ on the total Reynolds normal stresses are consistent with those on their LC-coherent part.

In the near-bottom region with $y^{+}<20$, the profiles of $\left\langle u^{T} u^{T}\right\rangle,\left\langle v^{T} v^{T}\right\rangle$ and $\left\langle w^{T} w^{T}\right\rangle$ are barely influenced by $k h$ (figure $21 a 3, b 3, c 3$ ), indicating that the interaction between LCs and residual turbulence is week and the residual turbulence is not significantly influenced by the reduction in LC intensity. However, because the LC-coherent motion makes a considerable contribution to $\left\langle u^{\prime} u^{\prime}\right\rangle$ and $\left\langle w^{\prime} w^{\prime}\right\rangle$ in the inner layer (figure 21a2,c2), the magnitudes of $\left\langle u^{\prime} u^{\prime}\right\rangle$ and $\left\langle w^{\prime} w^{\prime}\right\rangle$ decrease in the inner layer as the value of $k h$ increases. As shown in figures 21(a1) and 21(c1), the LC-coherent motion influences the profiles of $\left\langle u^{\prime} u^{\prime}\right\rangle$ and $\left\langle w^{\prime} w^{\prime}\right\rangle$ down to $y^{+}=10$ and $y^{+}=4$, respectively. Different from $\left\langle u^{L} u^{L}\right\rangle$ and $\left\langle w^{L} w^{L}\right\rangle$, the magnitude of $\left\langle v^{L} v^{L}\right\rangle$ is negligibly small in the inner layer below $y^{+}=100$ (figure 21b2), such that the effect of $k h$ on $\left\langle v^{\prime} v^{\prime}\right\rangle$ is consistent with that on $\left\langle v^{T} v^{T}\right\rangle$. As shown in figure $21(b 1, b 3)$, between $y^{+}=40$ and 100, the magnitudes of $\left\langle v^{\prime} v^{\prime}\right\rangle$ and $\left\langle v^{T} v^{T}\right\rangle$ both increase slightly as the value of $k h$ increases.

Figure 22 shows the effect of $L a_{t}$ on the profiles of the Reynolds normal stresses, their LC-coherent parts, and residual parts. Similar to the LC-coherent Reynolds shear stress discussed in $\$ 4.2$, the magnitudes of the LC-coherent Reynolds normal stresses are not monotonic with the increase of $L a_{t}$. As shown in figure 22(a2,b2,c2), the magnitudes of $\left\langle u^{L} u^{L}\right\rangle^{+},\left\langle v^{L} v^{L}\right\rangle^{+}$and $\left\langle w^{L} w^{L}\right\rangle^{+}$are all larger at $L a_{t}=0.7$ than at 0.4 and 0.9 , indicating that the intensity of the LCs reverses at $L a_{t}=0.7$. Further because the residual parts of the Reynolds normal stresses $\left\langle u^{T} u^{T}\right\rangle^{+}$and $\left\langle w^{T} w^{T}\right\rangle^{+}$ are not significantly influenced by the value of $L a_{t}$ (figure 22a3,c3), the magnitudes of $\left\langle u^{\prime} u^{\prime}\right\rangle^{+}$and $\left\langle w^{\prime} w^{\prime}\right\rangle^{+}$also reverse at $L a_{t}=0.7$ (figure 22a1,c1). In contrast, the magnitude of $\left\langle v^{\prime} v^{\prime}\right\rangle^{+}$is monotonic to the value increase of $L a_{t}$ (figure 22b1), due to the magnitude increase of $\left\langle v^{T} v^{T}\right\rangle^{+}$as the value of $L a_{t}$ decreases from 0.7 to 0.4 , which is also observed by Sinha et al. (2015) at $R e_{\tau}=395$.

\section{Budget of turbulence kinematic energy}

To further assess the effects of LCs on the energy transport, we study the budget of TKE. The transport equation of TKE reads

$$
\frac{\mathrm{D} k}{\mathrm{D} t}=P_{k}+T_{k}+\Pi_{k}+V_{k}+S_{k}+\varepsilon_{k} .
$$

Here, $k=\left\langle u_{i}^{\prime} u_{i}^{\prime}\right\rangle / 2$ is the TKE. The budget terms on the right-hand side are the production term $P_{k}$, turbulent diffusion term $T_{k}$, pressure diffusion term $\Pi_{k}$, viscous 

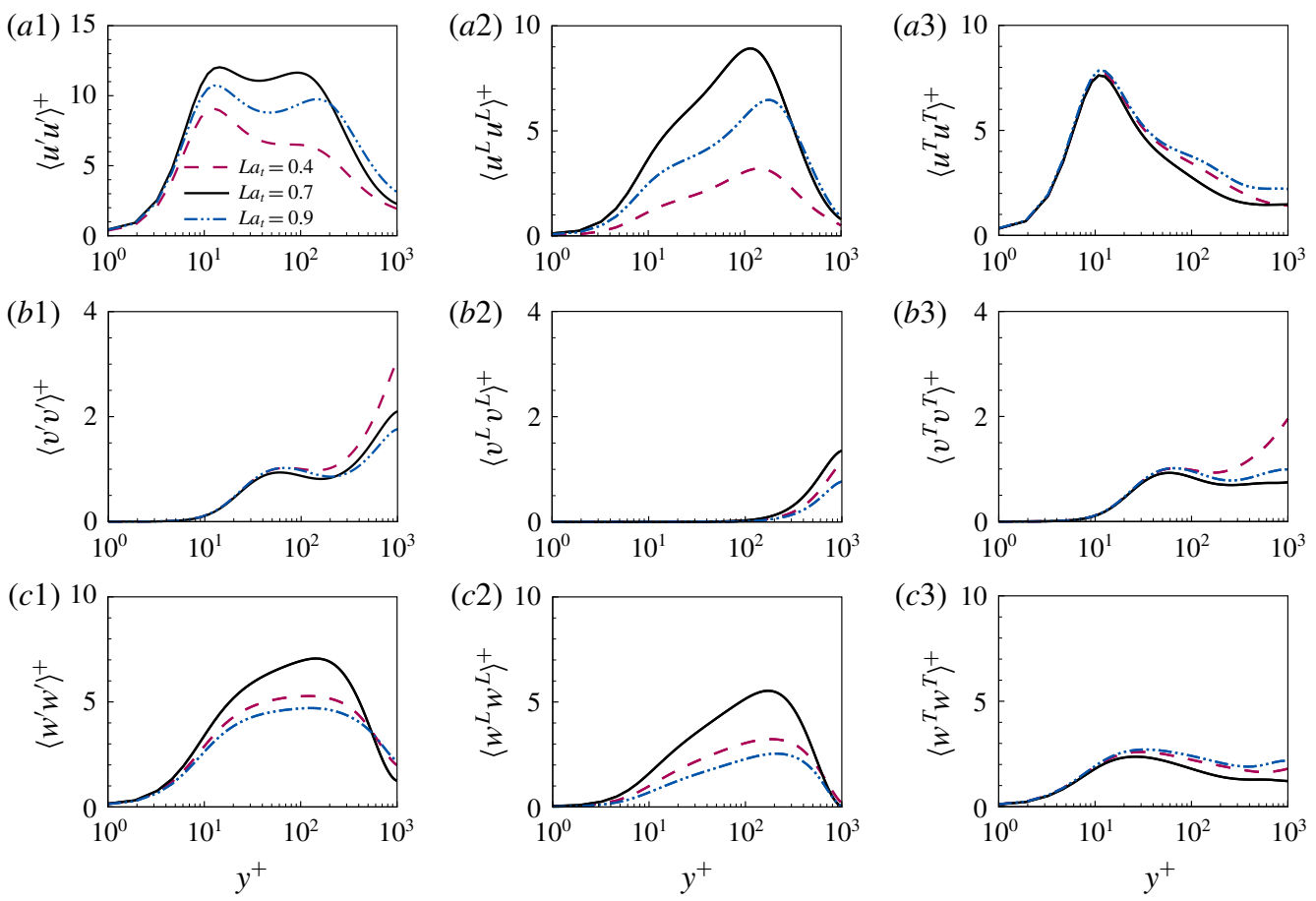

FIGURE 22. (Colour online) Profiles of $(a 1)\left\langle u^{\prime} u^{\prime}\right\rangle^{+}$, (a2) $\left\langle u^{L} u^{L}\right\rangle^{+}$, (a3) $\left\langle u^{T} u^{T}\right\rangle^{+}$, (b1) $\left\langle v^{\prime} v^{\prime}\right\rangle^{+}$, (b2) $\left\langle v^{L} v^{L}\right\rangle^{+}$, (b3) $\left\langle v^{T} v^{T}\right\rangle^{+}$, (c1) $\left\langle w^{\prime} w^{\prime}\right\rangle^{+}$, (c2) $\left\langle w^{L} w^{L}\right\rangle^{+}$and (c3) $\left\langle w^{T} w^{T}\right\rangle^{+}$in cases 1,7 and 8 with $L a_{t}=0.7,0.4$ and 0.9 , respectively.

and SGS diffusion term $V_{k}$, Stokes production term $S_{k}$ and viscous and SGS dissipation term $\varepsilon_{k}$, defined respectively as

$$
\begin{gathered}
P_{k}=-\left\langle u^{\prime} v^{\prime}\right\rangle \frac{\mathrm{d}\langle u\rangle}{\mathrm{d} y}, \\
T_{k}=-\frac{1}{2} \frac{\mathrm{d}\left\langle u_{i}^{\prime} u_{i}^{\prime} v^{\prime}\right\rangle}{\mathrm{d} y}, \\
\Pi_{k}=-\frac{1}{\rho} \frac{\mathrm{d}\left\langle p^{\prime} v^{\prime}\right\rangle}{\mathrm{d} y}, \\
V_{k}=v \frac{\mathrm{d}^{2} k}{\mathrm{~d} y^{2}}+\frac{\mathrm{d}\left\langle u_{i}^{\prime} \tau_{i 2}^{\prime s g s}\right\rangle}{\mathrm{d} y}, \\
S_{k}=-\left\langle u^{\prime} v^{\prime}\right\rangle \frac{\mathrm{d} u_{s}}{\mathrm{~d} y}, \\
\varepsilon_{k}=-v\left\langle\frac{\partial u_{i}^{\prime}}{\partial x_{j}} \frac{\partial u_{i}^{\prime}}{\partial x_{j}}\right\rangle-\left\langle\tau_{i j}^{\prime s g s} \frac{\partial u_{i}^{\prime}}{\partial x_{j}}\right\rangle .
\end{gathered}
$$

The above equations can also be found in McWilliams et al. (1997), Harcourt (2013) and Sinha et al. (2015).

The profiles of the budget terms of TKE pre-multiplied by $y$ are shown in figure 23 using the scaling of $u_{\tau}^{3}$, with a maximum balance error smaller than $1 \%$ of the dominant source terms. The pre-multiplication by $y$ makes the balance relationship 
(a)

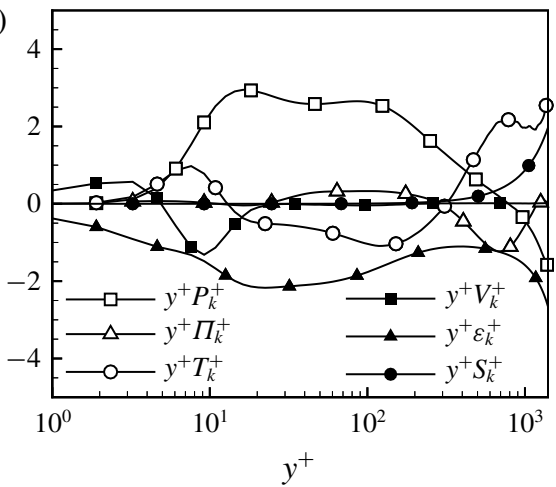

(b)

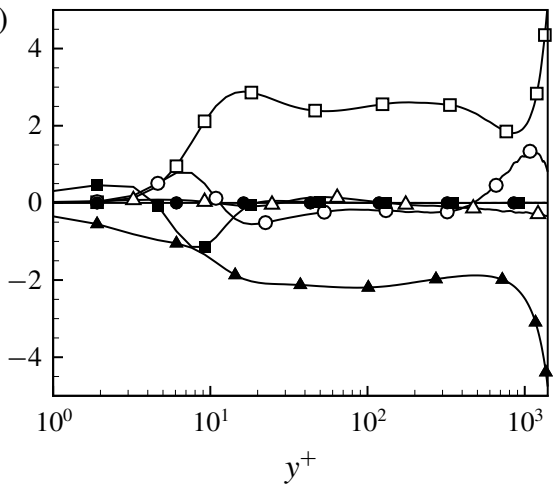

FIgURE 23. Pre-multiplied budget terms of TKE in $(a)$ Langmuir turbulence at $R e_{\tau}=$ 1000, $L a_{t}=0.7$ and $k h=0.5$ (case 1) and $(b)$ shear-driven turbulence at $R e_{\tau}=1000$ (case 9).

among the budget terms in the outer layer clear (Hoyas \& Jiménez 2008; Avsarkisov et al. 2014). From the comparison between figures 23(a) and 23(b), it is evident that below $y^{+}=100$, the balance relationship of budget terms in the shallow-water Langmuir turbulence is similar to that in the shear-driven turbulence. Above $y^{+}=10$, the energy gained from the production term $P_{k}$ is largely balanced by the viscous and SGS dissipation term $\varepsilon_{k}$. The turbulent diffusion term $T_{k}$ transports the energy above $y^{+}=10$ downward. The viscous diffusion term $V_{k}$ transports the energy in the buffer layer downward into the viscous sublayer to balance the dissipation term $\varepsilon_{k}$. The energy transport below $y^{+}=100$ shown in figure 23 is also similar to that in the canonical wall turbulence (Hoyas \& Jiménez 2008; Avsarkisov et al. 2014), indicating that the effect of LCs on the TKE budget is weak in the near-bottom region.

In the outer layer, the occurrence of LCs significantly alters the transport of TKE. In the Langmuir turbulence (figure 23a), there is a plateau region in the profile of $y^{+} P_{k}^{+}$between $y^{+}=15$ and 100. In the shear-driven turbulence (figure 23b), the plateau of $y^{+} P_{k}^{+}$extends to $y^{+}=300$. According to its definition (5.2), the value of $P_{k}^{+}$is determined by the Reynolds shear stress $-\left\langle u^{\prime} v^{\prime}\right\rangle^{+}$and the mean velocity gradient $\mathrm{d} u^{+} / \mathrm{d} y^{+}$. In the outer layer above $y^{+}=100$, the value of $-\left\langle u^{\prime} v^{\prime}\right\rangle^{+}$ is approximately unity in both Langmuir turbulence and shear-driven turbulence (figure $11 d$ ). As a result, the value of the pre-multiplied production term is approximately $y^{+} P_{k}^{+} \approx y^{+} \mathrm{d}\langle u\rangle^{+} / \mathrm{d} y^{+}=F$ (see (3.2) for the definition of $F$ ). In the Langmuir turbulence, due to the occurrence of LCs, the mean shear is reduced above the logarithmic layer, resulting in the value decrease of $F$ and $y^{+} P_{k}^{+}$above $y^{+}=100$. Above $y^{+}=400$, the major source of TKE changes from the production term $P_{k}$ to the turbulence diffusion term $T_{k}$ and Stokes production term $S_{k}$ in the Langmuir turbulence (figure $23 a$ ). It has been known that in the deep-water Langmuir turbulence, $S_{k}$ is an important source term in the transport equation of TKE, resulting in a large magnitude of vertical velocity fluctuations (Skyllingstad \& Denbo 1995; McWilliams et al. 1997; Li et al. 2005; Kukulka et al. 2010). In the following content, we first study the $S_{k}$ term to show the effect of Stokes drift of surface waves on the TKE budget in the shallow-water Langmuir turbulence.

Figure 24 compares the profiles of $S_{k} /\left(u_{\tau}^{3} / h\right)$ in cases $1-3$ with various Reynolds numbers. According to Harcourt (2013) and Suzuki \& Fox-Kemper (2016), it can be 

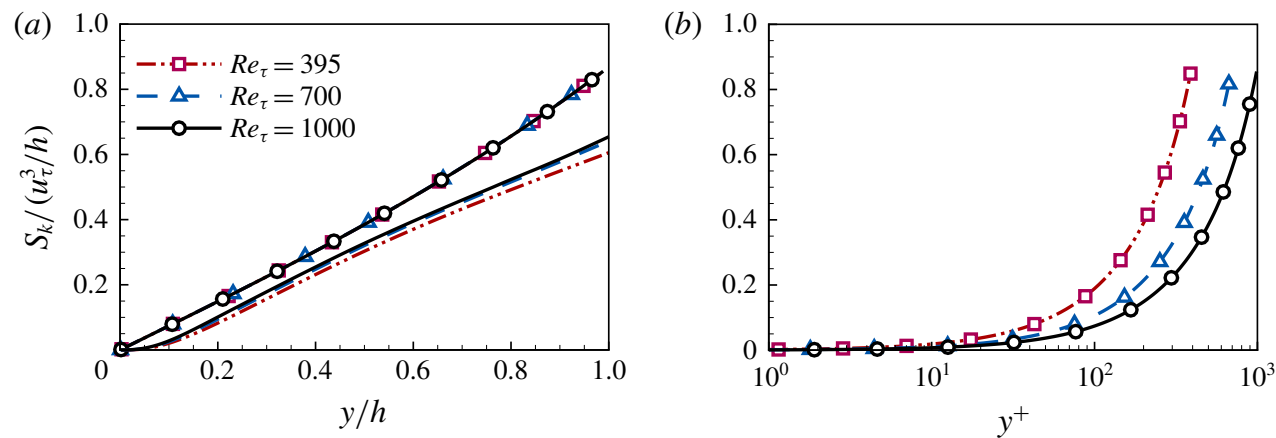

FIgURE 24. (Colour online) Profiles of the Stokes production term $S_{k}$ scaled by $u_{\tau}^{3} / h$ in the TKE budget in cases 1-3 with $R e_{\tau}=1000,700$ and 395, respectively, in $(a)$ watercolumn coordinate $y / h$ and $(b)$ near-bottom coordinate $y^{+}$. The lines without symbols in (a) are $S_{k}^{L}$.

derived that the Stokes production terms in the transport equations of $\left\langle u^{\prime} u^{\prime}\right\rangle,\left\langle v^{\prime} v^{\prime}\right\rangle$ and $\left\langle w^{\prime} w^{\prime}\right\rangle$ are respectively

$$
S_{11}=0, \quad S_{22}=2 S_{k}, \quad \text { and } \quad S_{33}=0 .
$$

Equation (5.8) indicates that the $S_{k}$ term is directly responsible for the enhancement of $\left\langle v^{\prime} v^{\prime}\right\rangle$ in the outer layer of the Langmuir turbulence (figure 16). This deduction can be further confirmed by the comparison between the profiles of $\left\langle v^{\prime} v^{\prime}\right\rangle$ and $S_{k}$. As shown in figures $16(a)$ and $24(a)$, above $y / h=0.2$, both $\left\langle v^{\prime} v^{\prime}\right\rangle$ and $S_{k}$ have large magnitudes, and their values increase fast as $y / h$ increases.

According to its definition (5.6), the $S_{k}$ term is determined by $-\left\langle u^{\prime} v^{\prime}\right\rangle$ and $\mathrm{d} u_{s} / \mathrm{d} y$. The value of $-\left\langle u^{\prime} v^{\prime}\right\rangle^{+}$is approximately unity in the outer layer (figure 11a), while $u_{s} / u_{\tau}$ is independent of the Reynolds number by its definition (2.4). As a result, the value of $S_{k} /\left(u_{\tau}^{3} / h\right)$ is expected to be insensitive to the Reynolds number, which is confirmed by figure 24(a). However, the effect of the Stokes production term on the LC-coherent fluctuations is slightly dependent on the Reynolds number. This can be explained using the decomposition $S_{k}=S_{k}^{L}+S_{k}^{T}$, where $S_{k}^{L}=-\left\langle u^{L} v^{L}\right\rangle \mathrm{d} u_{s} / \mathrm{d} y$ and $S_{k}^{T}=-\left\langle u^{T} v^{T}\right\rangle \mathrm{d} u_{s} / \mathrm{d} y$ are the Stokes productions of $\left\langle v^{L} v^{L}\right\rangle$ and $\left\langle v^{T} v^{T}\right\rangle$, respectively. The profiles of $S_{k}^{L}$ at various Reynolds numbers are also shown in figure 24(a). From the figure, it can be seen that the magnitude of $S_{k}^{L}$ increases slightly as the Reynolds number increases. Correspondingly, the magnitude of $\left\langle v^{L} v^{L}\right\rangle$ also increases with the Reynolds number in the water-column coordinate (figure 19a). On the other hand, as shown in figure 24(b), the profiles of $S_{k}$ shift to larger $y^{+}$as $\operatorname{Re}_{\tau}$ increases. In response, when observed in the near-bottom coordinate $y^{+}$, the effect of Stokes production on $\left\langle v^{\prime} v^{\prime}\right\rangle$ is weakened near the bottom. Therefore, as the Reynolds number increases, the profiles of $\left\langle v^{\prime} v^{\prime}\right\rangle$ below $y^{+}=50$ in the Langmuir turbulence approach that in the shear-driven turbulence (figure 16a).

Different from the Reynolds number effect, $k h$ and $L a_{t}$ affect the magnitude of $S_{k}$. Figure 25 shows the effects of $k h$ and $L a_{t}$ on the profile of $S_{k}$. It can be observed from the figure that as the value of either $k h$ or $L a_{t}$ increases, the magnitude of $S_{k}$ decreases. This is expected from the definitions of the $S_{k}$ term (5.6) and Stokes drift velocity (2.4). As a result of the reduction of $S_{k}$, the magnitude of $\left\langle v^{\prime} v^{\prime}\right\rangle$ also decreases in the outer layer (figures $21 b 1$ and 22b1), which leads to the suppression of vertical mixing. 

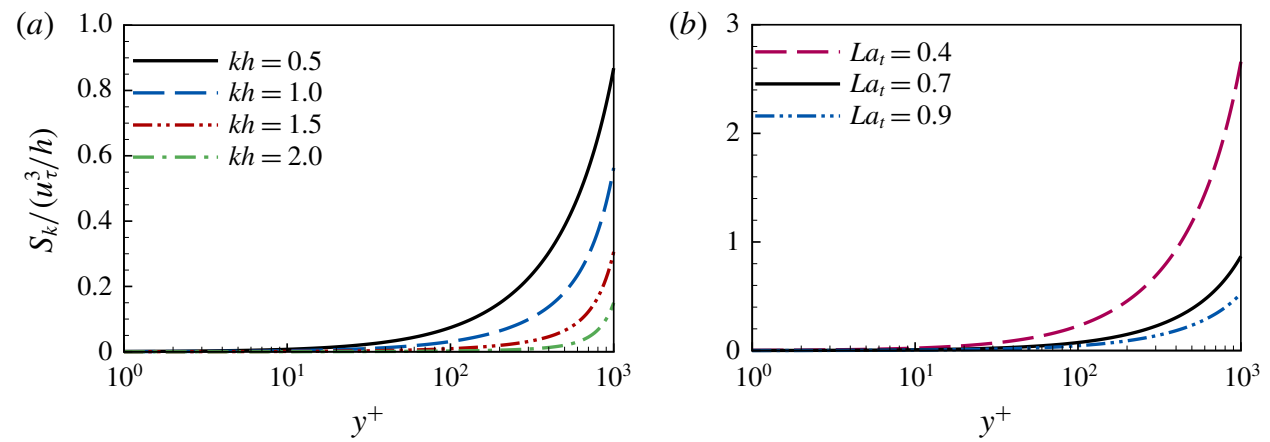

Figure 25. (Colour online) Profiles of the Stokes production term $S_{k}$ scaled by $u_{\tau}^{3} / h(a)$ in cases 1 and $4-6$ with $k h=0.5,1,1.5$ and 2 , respectively and $(b)$ cases 1,7 and 8 with $L a_{t}=0.7,0.4$ and 0.9 , respectively.

It is shown in figure 23 that the turbulence diffusion term $T_{k}$ is the most important source in the transport equation of TKE above $y^{+}=400$ in the Langmuir turbulence, and its magnitude is larger than that in the shear-driven turbulence. Here, we further study the effect of LCs on the $T_{k}$ term. The $T_{k}$ term can be decomposed as

$$
T_{k}=T_{k}^{T+}+T_{k}^{L *} .
$$

Here, the $T_{k}^{T+}$ term is the turbulent diffusion only related to the residual velocity fluctuations, defined as

$$
T_{k}^{T+}=-\frac{1}{2} \frac{\mathrm{d}\left\langle u_{i}^{T} u_{i}^{T} v^{T}\right\rangle}{\mathrm{d} y}
$$

The $T_{k}^{L *}$ term is defined as

$$
T_{k}^{L *}=\underbrace{-\frac{1}{2} \frac{\mathrm{d}\left\langle u_{i}^{L} u_{i}^{L} v^{L}+u_{i}^{T} u_{i}^{T} v^{L}\right\rangle}{\mathrm{d} y}}_{T_{k}^{L 1}} \underbrace{-\frac{\mathrm{d}\left\langle u_{i}^{L} u_{i}^{T} v^{T}\right\rangle}{\mathrm{d} y}}_{T_{k}^{L 2}},
$$

which includes the LC-coherent velocity fluctuations and represents the effect of the LCs. A similar triple decomposition was used by Martinat et al. (2014) to analyse the budget balance of $\left\langle u_{i}^{L} u_{i}^{L}\right\rangle / 2$ and $\left\langle u_{i}^{T} u_{i}^{T}\right\rangle / 2$ at $R e_{\tau}=395$. Figure 26 compares the profiles of $y^{+} T_{k}^{T+}, y^{+} T_{k}^{L *+}$ and $y^{+} T_{k}$ in Langmuir turbulence (case 1). As shown, above $y^{+}=200$, the magnitude of $y^{+} T_{k}^{L *+}$ is significantly larger than that of $y^{+} T_{k}^{T+}$, and the profiles of $y^{+} T_{k}^{L *+}$ and $y^{+} T_{k}^{+}$almost collapse. This indicates that the large magnitude of the turbulent diffusion term in the outer layer is contributed by the LCs.

According to (5.11), $T_{k}^{L *+}$ can be further decomposed into $T_{k}^{L 1+}$ and $T_{k}^{L 2+}$. The $T_{k}^{L 1+}$ term is the turbulence diffusion term induced by $v^{L}$, and $T_{k}^{L 2+}$ is the interaction between the residual turbulence and LCs. Figure 27 compares the profiles of $y^{+} T_{k}^{L *+}$, $y^{+} T_{k}^{L 1+}$ and $y^{+} T_{k}^{L 2+}$ in Langmuir turbulence (case 1). As shown, above $y^{+}=200$, the magnitude of $y^{+} T_{k}^{L 1+}$ is much larger than that of $y^{+} T_{k}^{L 2+}$, indicating that the large magnitude of $T_{k}^{L *+}$ and $T_{k}^{+}$in the outer layer is induced by the vertical mixing of the LCs. Therefore, the vertical velocity fluctuations of LCs generated by $S_{k}$ in the outer layer lead to the strong turbulent diffusion, which significantly alters the energy transport in the outer layer. 


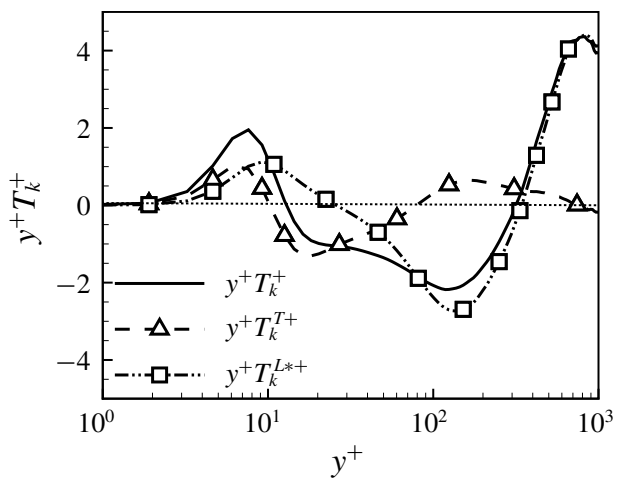

FIgURE 26. Profiles of pre-multiplied turbulent diffusion terms $y^{+} T_{k}^{+}, y^{+} T_{k}^{T+}$ and $y^{+} T_{k}^{L *+}$ in Langmuir turbulence (case 1 ).

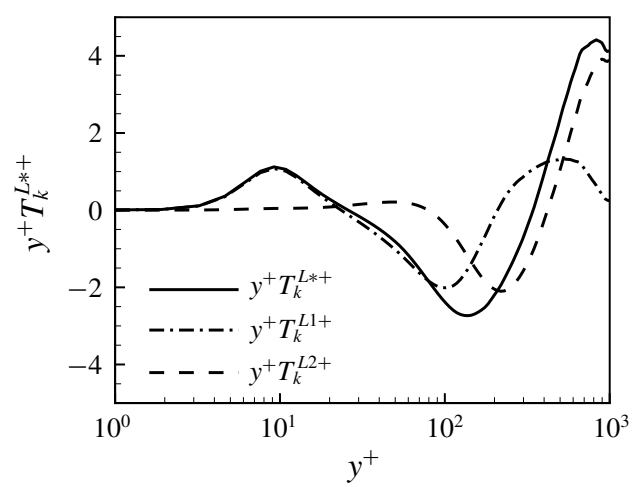

FIgURE 27. Profiles of pre-multiplied turbulent diffusion term $y^{+} T_{k}^{L *+}, y^{+} T_{k}^{L 1+}$ and $y^{+} T_{k}^{L 2+}$ in Langmuir turbulence (case 1).

\section{Summary}

In this study, wall-resolved large-eddy simulations based on the $\mathrm{C}-\mathrm{L}$ equations have been performed to investigate the influence of LCs on the turbulence in the bottom boundary layer of shallow water. The effect of Reynolds number $R e_{\tau}$ on the mean velocity and Reynolds stresses is investigated by varying $R e_{\tau}$ from 395 up to 1000 . The wall-resolved LES of the shallow-water Langmuir turbulence at $\operatorname{Re}_{\tau}=1000$ is carried out for the first time. The effects of the dimensionless wavenumber of surface waves $k h$ and turbulent Langmuir number $L a_{t}$, two parameters dominating the intensity of LCs, are also studied. To analyse the result, the total velocity fluctuation $u_{i}^{\prime}$ is decomposed into the LC-coherent velocity fluctuation $u_{i}^{L}$ and residual turbulent velocity fluctuation $u_{i}^{T}$, and their contributions to the Reynolds stresses are analysed. The transport equations of TKE are studied to further reveal the effect of LCs on the energy transport. The major findings of this paper are summarized as follows.

In the analysis of the mean streamwise velocity, we focus on the effects of $R e, k h$ and $L a_{t}$ on the thickness of the logarithmic layer, identified as the plateau region in the profile of the diagnostic function $F$. The present simulations show that the logarithmic layer is absent at low Reynolds numbers $R e_{\tau}=395$ and 700 , but reappears at $R e_{\tau}=$ 1000. As the value of either $k h$ or $L a_{t}$ increases, the magnitude of the $\mathrm{C}-\mathrm{L}$ vortex 
force in the momentum equation decreases, leading to the thickness increase of the logarithmic layer. In the plateau region of $F$, the value of $F$ is identical to the von Kármán constant. It is observed that the von Kármán constant in the bottom boundary of the Langmuir turbulence under investigation varies in a narrow range with $0.362 \pm$ 0.003 , which is below the classic value 0.41 .

In the outer layer, the LC-coherent part makes important contributions to the total Reynolds stresses. In the water-column coordinate $y / h$, the peak locations of $\left\langle-u^{L} v^{L}\right\rangle$, $\left\langle u^{L} u^{L}\right\rangle$ and $\left\langle w^{L} w^{L}\right\rangle$ remain the same at various $R e_{\tau}$ with given values of $L a_{t}$ and $k h$, indicating that the locations of LCs are scaled by $h$. On the other hand, when observed in the near-bottom coordinate $y^{+}$, the profile of $\left\langle-u^{L} v^{L}\right\rangle$ shifts to larger $y^{+}$ at higher Reynolds number, indicating a weaker influence of LCs on the momentum transfer near the bottom, which results in the reappearance of the logarithmic layer at $R e_{\tau}=1000$. Furthermore, as the Reynolds number increases, the peak location of $\left\langle u^{T} u^{T}\right\rangle$ remains unchanged, while that of $\left\langle u^{L} u^{L}\right\rangle$ shifts to larger $y^{+}$. As a result, the peaks of both $\left\langle u^{T} u^{T}\right\rangle$ and $\left\langle u^{L} u^{L}\right\rangle$ are shown in the profile of $\left\langle u^{\prime} u^{\prime}\right\rangle$ at $R e_{\tau}=700$ and 1000 , resulting in a bimodal shape. The profile of $\left\langle w^{\prime} w^{\prime}\right\rangle$ only has a single peak, with its location close to the peak of $\left\langle w^{L} w^{L}\right\rangle$. Owing to the contribution of LCs, the magnitude of $\left\langle v^{\prime} v^{\prime}\right\rangle$ increases fast with $y / h$ in the outer layer above $y / h=0.17$ at all of the Reynolds numbers under investigation.

As the value of $k h$ increases, the intensity of LCs is reduced, characterized by the magnitude decrease of $\left\langle-u^{L} v^{L}\right\rangle,\left\langle u^{L} u^{L}\right\rangle,\left\langle v^{L} v^{L}\right\rangle$ and $\left\langle w^{L} w^{L}\right\rangle$ in the outer layer, which leads to the magnitude decrease of their corresponding total Reynolds stresses. Furthermore, at larger value of $k h$, the peak locations of $-\left\langle u^{L} v^{L}\right\rangle,\left\langle u^{L} u^{L}\right\rangle$ and $\left\langle w^{L} w^{L}\right\rangle$ are farther away from the bottom, indicating a weaker influence of LCs on the momentum and energy transfer in the bottom inner layer. As a result, the thickness of the logarithmic layer increases as the value of $k h$ increases.

The intensity of LCs is not monotonic in response to the increase of $L a_{t}$. The magnitudes of $-\left\langle u^{L} v^{L}\right\rangle,\left\langle u^{L} u^{L}\right\rangle,\left\langle v^{L} v^{L}\right\rangle$ and $\left\langle w^{L} w^{L}\right\rangle$ all reverse at $L a_{t}=0.7$. As the value of $L a_{t}$ decreases from 0.9 to 0.7 , the logarithmic layer becomes thinner because of the stronger effect of LCs on the momentum transfer. As $L a_{t}$ further decreases from 0.7 to 0.4 , although the LCs become weaker, the logarithmic layer is thinner at $L a_{t}=0.4$ due to the enhancement of outer-layer large-scale turbulence motions that penetrate to the near-bottom region. This effect alters the momentum transfer in the near-bottom region, and tends to disrupt the logarithmic law.

The effects of $R e_{\tau}, k h$ and $L a_{t}$ on the intensity of LCs are further explained through the analyses of the Stokes production term $S_{k}$ in the transport equation of TKE. The Stokes production term induces large magnitude of vertical velocity fluctuations in the outer layer mainly contributed by LCs. The contribution of the Stoke production term to LCs, $S_{k}^{L}$, is slightly dependent on $R e_{\tau}$, so that $\left\langle v^{L} v^{L}\right\rangle$ varies with $R e_{\tau}$. As the value of either $k h$ or $L a_{t}$ increases, the magnitude of $S_{k}$ decreases, leading to the smaller magnitude of $\left\langle v^{\prime} v^{\prime}\right\rangle$ in the outer layer. Due to the occurrence of LCs, the turbulence diffusion term $T_{k}$ is significantly amplified in the outer layer of the Langmuir turbulence, which becomes the most important source of TKE instead of the production term $P_{k}$ as in the shear-driven turbulence.

Finally, we remark that the present results show the capability of numerical simulations in the investigation of flow details of the bottom boundary layer in shallow-water Langmuir turbulence. Before we can reach a much higher Reynolds number of practical interest with wall-modelled LES, it is important to examine the logarithmic law using wall-resolved LES. Our LES results show that the disruption effect of LCs on the logarithmic law becomes less significant as the Reynolds 
number increases and the logarithmic layer reappears at $R e_{\tau}=1000$, indicating that the wall-layer modelling based on the logarithmic law can still be used at high Reynolds number. Recently, Golshan et al. (2017) conducted wall-modelled LES with the conventional wall-layer model based on the logarithmic law, and captured the disruption of the logarithmic layer in the profile of the mean velocity at $R e_{\tau}=395$. In the future, it will be of interest to examine if the wall-layer model based on the logarithmic law can capture the reappearance of the logarithmic layer and the thinning effect of LCs on the logarithmic layer at $R e_{\tau}=1000$ and higher. However, care must be taken to ensure that the first grid is located in the logarithmic layer, given that the logarithmic layer is thinner in the Langmuir turbulence than in shear-driven turbulence.

\section{Acknowledgements}

This work is partially supported by the Office of Naval Research, National Science Foundation and Minnesota Sea Grant.

\section{REFERENCES}

AfZAL, N. \& YAJNIK, K. 1973 Analysis of turbulent pipe and channel flows at moderately large Reynolds number. J. Fluid Mech. 61, 23-31.

Akan, C., Tejada-Martínez, A. E., Grosch, C. E. \& Martinat, G. 2013 Scalar transport in large-eddy simulation of Langmuir turbulence in shallow water. Cont. Shelf Res. 55, 1-16.

Alfredsson, P. H., Segalini, A.\& ÖRlÜ, R. 2011 A new scaling for the streamwise turbulence intensity in wall-bounded turbulent flows and what it tells us about the 'outer' peak. Phys. Fluids 23, 041702.

Andrews, D. G. \& MCInTyRe, M. E. 1978 An exact theory of nonlinear waves on a Lagrangianmean flow. J. Fluid Mech. 89, 609-646.

Assaf, G., Gerard, R. \& Gordon, A. L. 1971 Some mechanisms of oceanic mixing revealed in aerial photographs. J. Geophys. Res. Oceans 76, 6550-6572.

Avsarkisov, V., Hoyas, S., Oberlack, M.\& Garcia-Galache, J. P. 2014 Turbulent plane Couette flow at moderately high Reynolds number. J. Fluid Mech. 751, R1.

Bernardini, M., Pirozzoli, S. \& ORlandi, P. 2014 Velocity statistics in turbulent channel flow up to $R e_{\tau}=4000$. J. Fluid Mech. 742, 171-191.

BlACKWELDER, R. F. \& ECKELMANN, H. 1979 Streamwise vortices associated with the bursting phenomenon. J. Fluid Mech. 94, 577-594.

CAвот, W. \& Moin, P. 2000 Approximate wall boundary conditions in the large-eddy simulation of high Reynolds number flow. Flow Turbul. Combust. 63, 269-291.

CHAPMAN, D. R. 1979 Computational aerodynamics development and outlook. AIAA J. 17, 1293-1313.

CHOI, H. \& MoIN, P. 2012 Grid-point requirements for large eddy simulation: Chapman's estimates revisited. Phys. Fluids 24, 011702.

CraIK, A. D. D. 1977 The generation of Langmuir circulations by an instability mechanism. J. Fluid Mech. 81, 209-223.

Craik, A. D. D. \& Leibovich, S. 1976 A rational model for Langmuir circulations. J. Fluid Mech. 73, 401-426.

D'Asaro, E. A. 2014 Turbulence in the upper-ocean mixed layer. Annu. Rev. Mar. Sci 6, 101-115.

DethlefF, D. \& Kemepema, E. W. 2007 Langmuir circulation driving sediment entrainment into newly formed ice: tank experiment results with application to nature (Lake Hattie, United States; Kara Sea, Siberia). J. Geophys. Res. Oceans 112, C02004.

Dierssen, H. M., Zimmerman, R. C., Drake, L. A. \& Burdige, D. J. 2009 Potential export of unattached benthic macroalgae to the deep sea through wind-driven Langmuir circulation. Geophys. Res. Lett. 36, L04602. 
FAller, A. J. \& CAPONI, E. A. 1978 Laboratory studies of wind-driven Langmuir circulations. J. Geophys. Res. Oceans 83, 3617-3633.

FARmer, D. \& Li, M. 1995 Patterns of bubble clouds organized by Langmuir circulation. J. Phys. Oceanogr. 25, 1426-1440.

Gargett, A., Wells, J., Tejada-Martínez, A. E. \& Grosch, C. E. 2004 Langmuir supercells: a mechanism for sediment resuspension and transport in shallow seas. Science 306, 1925-1928.

Gargett, A. E. \& Grosch, C. E. 2014 Turbulence process domination under the combined forcings of wind stress, the Langmuir vortex force, and surface cooling. J. Phys. Oceanogr. 44 (1), 44-67.

Gargett, A. E., Savidge, D. K. \& Wells, J. R. 2014 Anatomy of a Langmuir supercell event. J. Mar. Res. 72 (3), 127-163.

Gargett, A. E. \& Wells, J. R. 2007 Langmuir turbulence in shallow water. Part 1. Observations. J. Fluid Mech. 576, 27-61.

Germano, M., Piomelli, U., Moin, P. \& CABot, W. H. 1991 A dynamic subgrid-scale eddy viscosity model. Phys. Fluids 3, 1760-1765.

Golshan, R., Tejada-Martínez, A. E., Juha, M. J. \& Bazilevs, Y. 2017 LES and RANS simulation of wind-and wave-forced oceanic turbulent boundary layers in shallow water with wall modeling. Comput. Fluids 142, 96-108.

Grosch, C. E. \& GARgetT, A. E. 2016 Why do LES of Langmuir supercells not include rotation? J. Phys. Oceanogr. 46 (12), 3595-3597.

Harcourt, R. R. 2013 A second-moment closure model of Langmuir turbulence. J. Phys. Oceanogr. 43 (4), 675-697.

HARCourt, R. R. \& D'Asaro, E. A. 2008 Large-eddy simulation of Langmuir turbulence in pure wind seas. J. Phys. Oceanogr. 38, 1542-1562.

Holm, D. D. 1996 The ideal Craik-Leibovich equations. Physica D 98, 415-441.

HoYAS, S. \& JimÉnEZ, J. 2006 Scaling of the velocity fluctuations in turbulent channels up to $R e_{\tau}=2003$. Phys. Fluids 18, 011702.

HoyAS, S. \& JimÉnEZ, J. 2008 Reynolds number effects on the Reynolds-stress budgets in turbulent channels. Phys. Fluids 20, 101511.

JeOng, J., Hussain, F., Schoppa, W. \& Kim, J. 1997 Coherent structures near the wall in a turbulent channel flow. J. Fluid Mech. 332, 185-214.

JimÉneZ, J. 2012 Cascades in wall-bounded turbulence. Annu. Rev. Fluid Mech. 44, 27-45.

JimÉneZ, J. \& MOSER, R. D. 2007 What are we learning from simulating wall turbulence? Proc. R. Soc. Lond. A 365, 715-732.

JimÉnez, J. \& Pinelli, A. 1999 The autonomous cycle of near-wall turbulence. J. Fluid Mech. 389, $335-359$.

Kemp, P. H. \& Simons, R. R. 1982 The interaction between waves and a turbulent current: waves propagating with the current. J. Fluid Mech. 116, 227-250.

KIM, J. \& MoIN, P. 1985 Application of a fractional-step method to incompressible Navier-Stokes equations. J. Comput. Phys. 59, 308-323.

Kim, J., Moin, P. \& Moser, R. 1987 Turbulence statistics in fully developed channel flow at low Reynolds number. J. Fluid Mech. 177, 133-166.

KLEWICKI, J., FIfE, P. \& WeI, T. 2009 On the logarithmic mean profile. J. Fluid Mech. 638, 73-93.

Kukulka, T., Plueddemann, A. J. \& Sullivan, P. P. 2012 Nonlocal transport due to Langmuir circulation in a coastal ocean. J. Geophys. Res. Oceans 117, C12007.

Kukulka, T., Plueddemann, A. J., Trowbridge, J. H. \& Sullivan, P. P. 2010 Rapid mixed layer deepening by the combination of Langmuir and shear instabilities: a case study. J. Phys. Oceanogr. 40, 2381-2400.

Kukulka, T., Plueddemann, A. J., Trowbridge, J. H. \& Sullivan, P. P. 2011 The influence of crosswind tidal currents on Langmuir circulation in a shallow ocean. J. Geophys. Res. Oceans 116, C08005.

LANGMUiR, I. 1938 Surface motion of water induced by wind. Science 87, 119-123.

Lee, M. \& Moser, R. D. 2015 Direct numerical simulation of turbulent channel flow up to $R e_{\tau}=$ 5200. J. Fluid Mech. 774, 395-415. 
Leibovich, S. 1977 On the evolution of the system of wind drift currents and Langmuir circulations in the ocean. Part 1. Theory and averaged current. J. Fluid Mech. 79, 715-743.

Leibovich, S. 1980 On wave-current interaction theories of Langmuir circulations. J. Fluid Mech. 99, 715-724.

Leibovich, S. 1983 The form and dynamics of Langmuir circulations. Annu. Rev. Fluid Mech. 15, 391-427.

Li, M., Garrett, C. \& Skyllingstad, E. 2005 A regime diagram for classifying turbulent large eddies in the upper ocean. Deep Sea Res. I 52, 259-278.

Lilly, D. K. 1992 A proposed modification of the Germano subgrid-scale closure method. Phys. Fluids 4, 633-635.

Longuet-Higgins, M. S. 1953 Mass transport in water waves. Proc. R. Soc. Lond. A 245, 535-581.

LUChIni, P. 2017 Universality of the turbulent velocity profile. Phys. Rev. Lett. 118, 224501.

Manna, M., VacCA, A. \& Verzicco, R. 2012 Pulsating pipe flow with large-amplitude oscillations in the very high frequency regime. Part 1. Time-averaged analysis. J. Fluid Mech. 700, 246-282.

Martinat, G., Grosch, C. E. \& Gatski, T. B. 2014 Modeling of Langmuir circulation: triple decomposition of the Craik-Leibovich model. Flow Turbul. Combust. 92, 395-411.

Martinat, G., Xu, Y., Grosch, C. E. \& Tejada-Martínez, A. E. 2011 LES of turbulent surface shear stress and pressure-gradient-driven flow on shallow continental shelves. Ocean Dyn. 61 (9), 1369-1390.

Marusic, I., Mathis, R. \& Hutchins, N. 2010 High Reynolds number effects in wall turbulence. Intl J. Heat Fluid Flow 31, 418-428.

Marusic, I., Monty, J. P., Hultmark, M. \& Smiths, A. J. 2013 On the logarithmic region in wall turbulence. J. Fluid Mech. 716, R3.

McWilliams, J. C., Huckle, E., Liang, J. \& Sullivan, P. P. 2014 Langmuir turbulence in swell. J. Phys. Oceanogr. 44, 870-890.

McWilliams, J. C., Restrepo, J. M. \& Lane, E. M. 2004 An asymptotic theory for the interaction of waves and currents in coastal waters. J. Fluid Mech. 511, 135-178.

McWilliams, J. C., Sullivan, P. P. \& Moeng, C. H. 1997 Langmuir turbulence in the ocean. J. Fluid Mech. 334, 1-30.

Millikan, C. B. 1938 A critical discussion of turbulent flows in channels and circular tubes. In Proceedings of the 5th International Congress on Applied Mechanics, Wiley.

Mizuno, Y. \& JiMÉnEZ, J. 2011 Mean velocity and length-scales in the overlap region of wallbounded turbulent flows. Phys. Fluids 23, 085112.

Moser, R. D., Kim, J. \& Mansour, N. N. 1999 Direct numerical simulation of turbulent channel flow up to $R e_{\tau}=590$. Phys. Fluids 11, 943-945.

Nagib, H. M. \& Chauhan, K. A. 2008 Variations of von Kármán coefficient in canonical flows. Phys. Fluids 20, 101518.

NePf, H. M. 1992 The production and mixing effects of Langmuir circulations. PhD thesis, Department of Civil Engineering, Stanford University.

NoH, Y., Min, H. S. \& RAASCH, S. 2004 Large eddy simulation of the ocean mixed layer: the effects of wave breaking and Langmuir circulation. J. Phys. Oceanogr. 34, 720-735.

PAnton, R. L. 2001 Overview of the self-sustaining mechanisms of wall turbulence. Prog. Aerosp. Sci. 37, 341-383.

PhilliPs, O. M. 1967 The Dynamics of the Upper Ocean. Cambridge University Press.

Piomelli, U. \& Balaras, E. 2002 Wall-layer models for large-eddy simulations. Annu. Rev. Fluid Mech. 34, 349-374.

POLlaRD, R. T. 1977 Observations and theolies of Langmuir circulations and their role in near surface mixing. In A Voyage of Discovery: George Deacon 70th Anniversary Volume, pp. 235-251. Pergamon Press.

RAmaprian, B. R. \& TU, S. W. 1983 Fully developed periodic turbulent pipe flow. Part 2. The detailed structure of the flow. J. Fluid Mech. 137, 59-81.

Robinson, S. K. 1991 Coherent motions in the turbulent boundary layer. Annu. Rev. Fluid Mech. 23 (1), 601-639. 
RotTa, J. 1962 Turbulent boundary layers in incompressible flow. Prog. Aerosp. Sci. 2, 1-95.

SAVIDGE, D. K. \& GARgetT, A. E. 2017 Langmuir supercells on the middle shelf of the South Atlantic Bight: 1. Cell structure. J. Mar. Res. 75 (2), 49-79.

Scagliarini, A., Einarsson, H., Gylfason, Á. \& Toschi, F. 2015 Law of the wall in an unstably stratified turbulent channel flow. J. Fluid Mech. 781, R5.

Scott, J. T., Myer, G. E., Stewart, R. \& Walther, E. G. 1969 On the mechanism of Langmuir circulations and their role in epilimnion mixing. Limnol. Oceanogr. 14, 493-503.

Scotti, A. \& Piomelli, U. 2001 Numerical simulation of pulsating turbulent channel flow. Phys. Fluids 13, 1367-1384.

Sinha, N., Tejada-Martínez, A. E., Akan, C. \& Grosch, C. E. 2015 Toward a K-profile parameterization of Langmuir turbulence in shallow coastal shelves. J. Phys. Oceanogr. 45 (12), 2869-2895.

Skyllingstad, E. D. \& Denbo, D. W. 1995 An ocean large eddy simulation of Langmuir circulations and convection in the surface mixed layer. J. Geophys. Res. Oceans 100, $8501-8522$.

Smith, J., Pinkel, R. \& Weller, R. A. 1987 Velocity structure in the mixed layer during MiLDEX. J. Phys. Oceanogr. 17, 425-439.

Smith, J. A. 2001 Observations and theories of Langmuir circulation: a story of mixing. In Fluid Mechanics and the Environment: Dynamical Approaches, pp. 295-314. Springer.

Sullivan, P. P. \& MCWilliams, J. C. 2010 Dynamics of winds and currents coupled to surface waves. Аnnu. Rev. Fluid Mech. 42, 19-42.

Sullivan, P. P., McWilliams, J. C. \& Melville, W. K. 2007 Surface gravity wave effects in the oceanic boundary layer: large-eddy simulation with vortex force and stochastic breakers. J. Fluid Mech. 593, 405-452.

Sullivan, P. P., Romero, L., McWilliams, J. C. \& Melville, W. K. 2012 Transient evolution of Langmuir turbulence in ocean boundary layers driven by hurricane winds and waves. J. Phys. Oceanogr. 42, 1959-1980.

SuZUKI, N. \& FoX-Kemper, B. 2016 Understanding Stokes forces in the wave-averaged equations. J. Geophys. Res. Oceans 121 (5), 3579-3596.

Tejada-Martínez, A. E., Akan, C., Sinha, N., Grosch, C. E. \& Martinat, G. 2013 Surface dynamics in LES of full-depth Langmuir circulation in shallow water. Phys. Scr. 2013, 014008.

Tejada-Martínez, A. E. \& Grosch, C. E. 2007 Langmuir turbulence in shallow water. Part 2. Large-eddy simulation. J. Fluid Mech. 576, 63-108.

Tejada-Martínez, A. E., Grosch, C. E., Sinha, N., Akan, C. \& Martinat, G. 2012 Disruption of the bottom $\log$ layer in large-eddy simulations of full-depth Langmuir circulation. J. Fluid Mech. 699, 79-93.

Thorpe, S. A. 1992 The breakup of Langmuir circulation and the instability of an array of vortices. J. Phys. Oceanogr. 22, 350-360.

Thorpe, S. A. 2004 Langmuir circulation. Annu. Rev. Fluid Mech. 36, 55-79.

Townsend, A. A. 1976 The Structure of Turbulent Shear Flow. Cambridge University Press.

Walker, R., Tejada-Martínez, A. E. \& Grosch, C. E. 2016 Large-eddy simulation of a coastal ocean under the combined effects of surface heat fluxes and full-depth Langmuir circulation. J. Phys. Oceanogr. 46 (8), 2411-2436.

Weller, R. A. \& PriCE, J. F. 1988 Langmuir circulation within the oceanic mixed layer. Deep Sea Res. I 35, 711-747.

Zagarola, M. V., Perry, A. E. \& Smits, A. J. 1997 Log laws or power laws: the scaling in the overlap region. Phys. Fluids 9, 2094-2100. 